\title{
Ageostrophic contribution by the wind and waves induced flow to the lateral stirring in the Mediterranean Sea
} Orfila, ${ }^{\mathrm{d}}$

${ }^{\text {a } U n i v e r s i t y ~ o f ~ T o u l o n ~-~ M e d i t e r r a n e a n ~ I n s t i t u t e ~ o f ~ O c e a n o g r a p h y ~(M I O), B a ̂ t i m e n t ~ X, ~} 83130$ La Garde, France

b SOCIB, Balearic Islands Coastal Observing System, 07007 Palma de Mallorca, Balearic Islands, Spain

${ }^{\mathrm{c}}$ Brown University, Department of Earth, Environmental, and Planetary Sciences, 02912 Providence, Rhode Island, USA

${ }^{\mathrm{d}}$ Mediterranean Institute for Advanced Studies (CSIC-UIB), 07190 Esporles, Balearic Islands, Spain 
ABSTRACT: We study the impact of the Ekman currents and Stokes drift on the horizontal mixing and transport properties of the Mediterranean Sea. FSLE at the ocean surface are computed over the whole basin using 25 years of satellite altimetry derived geostrophic currents, $10-\mathrm{m}$ wind velocity and wave fields. We find that the transport pathways unveiled by the geostrophic Lagrangian Coherent Structures (LCS) are significantly modified by the ageostrophic currents (i.e. Ekman and Stokes induced velocities), often leading to a decrease of the retention capacity of the eddies. An exhaustive assessment of the regional dependence and temporal variability of the FSLE shows an increase of the horizontal mixing activity, due to the ageostrophic component, up to $37 \%$ in regions such as the Gulf of Lion or the Aegean Sea, during the seasons where wind and waves are intense and persistent. Positive trends in the total FSLE (up to 1.2\% of the value of FSLE per year in some regions) suggest that Mediterranean Sea has experienced a significant increase in mixing activity over the last decades. Ageostrophic features are considered to play a role in determining the properties of the relative dispersion. Through the analysis of the Lagrangian Anisotropy Index (LAI) using virtual and real pair of drifters, we observe that the particle dispersion is mainly dominated by the zonal flow, and that the ageostrophic currents induce meridional dispersion, particularly in regions where wind and wave are intensified.

This work has been submitted to Journal of Physical Oceanography. Copyright in this work may be transferred without further notice. The paper is a non-peer reviewed preprint submitted to EarthArXiv 


\section{Introduction}

Accurate assessment of surface velocities is fundamental for the analysis of the energy budgets at the ocean interface, as well as, for the measurement and subsequent prediction of fluid particle motions in the ocean, with implications in activities such as the mitigation of oil spills (Abascal et al. 2009; Sayol et al. 2014), the fate of marine debris (Onink et al. 2019), or to determine the connectivity patterns among different ocean regions (Rossi et al. 2014; Ser-Giacomi et al. 2021), among many others. In particular, transport and mixing properties in the upper layers have profound consequences on the biogeochemical cycles and the dynamics of marine species, whose knowledge is therefore crucial to understand the mechanisms regulating marine ecosystem (Lévy et al. 2018; Legrand et al. 2019; Hernández-Carrasco et al. 2020).

Ocean dynamics is mainly driven by the geostrophic currents and, up to a significant extent, modulated by the wind-driven Ekman velocities and the wave-induced Stokes drift (Polton et al. 2005; McWilliams and Restrepo 1999; Suzuki and Fox-Kemper 2016). These components contribute to the variability of the dynamics at the upper ocean layers with different spatial and temporal scales. While geostrophic currents are related to mesoscale and slow processes, the ageostrophy associated with wind and waves, induces high-frequency modifications to the large scale motions (Hui and Xu 2016; Morales-Márquez et al. 2020).

Several works have analyzed the contribution of wind and waves on surface currents (Hui and Xu 2016; Onink et al. 2019). The first attempt to understand the ageostrophic component at the ocean surface by the wind stress was developed more than a century ago through the Ekman's seminal paper (Ekman 1905). Since then, many works improved the classical Ekman model modifying the parameterization of the vertical structure of the wind forcing through different eddy viscosity profiles (Welander 1957; Price et al. 1987; Wenegrat and McPhaden 2016) or including the effects of waves (Huang 1979; Polton et al. 2005; McWilliams et al. 2012). Previous studies using an expression for the total surface currents as a linear combination of both geostrophic and ageostrophic (Ekman and Stokes) components based on available observations, have shown the important role of the wind and wave induced velocities in the global surface dynamics (Sudre et al. 2013; Ardhuin et al. 2009; Hui and Xu 2016).

The growing evidence that Ekman and Stokes velocity components have a strong impact on surface ocean dynamics has raised increasing interest in better understanding the effect of these 
ageostrophic currents on the fate of transported particles (Onink et al. 2019; Dobler et al. 2019). Previous studies have pointed up that Ekman currents play an important role in the accumulation of microplastics in the main subtropical ocean gyres (Onink et al. 2019). Indeed, the waveinduced velocity dramatically affects the direction of the Lagrangian trajectories of marine debris advected by the geostrophic currents in the southern Indian basin (Dobler et al. 2019). The precise contribution of each velocity component on the lateral stirring and relative dispersion remains unknown. The Lagrangian dynamics of the ocean flow can be readily explored by the Finite Size Lyapunov Exponents (FSLE) (Hernández-Carrasco et al. 2011). FSLE is the usual method used to analyze the dispersion properties of the turbulent flow (Lacorata et al. 2001), as well as, to reveal relevant spatial structures in ocean flows (d'Ovidio et al. 2004), i.e. the Lagrangian Coherent Structures (LCS). LCS strongly organize the transport in a dynamical fluid system (see Haller (2015) for a review). The importance of the LCS in the structuring of the biogechemical properties and ocean ecosystems have been largely demonstrated in previous studies. For example, the LCS obtained from ridges of FSLE have been correlated with filaments of remote-sensed chlorophyll (Chl $a$ ) (Lehahn et al. 2007; Hernández-Carrasco et al. 2018, 2020), primary production (Hernández-Carrasco et al. 2014), sea bird foraging behavior (Kai et al. 2009), and with the modelled extension of oxygen minimum zones (Bettencourt et al. 2015).

In this work we analyze the impact of the Ekman and Stokes components on the geostrophic LCS and on dispersion properties at the ocean surface in the Mediterranean Sea. Recently, MoralesMárquez et al. (2020) by solving the momentum equation in the steady state for wind and waves (Polton et al. 2005), presented a regionalization of the Mediterranean Sea surface dynamics as a function of the relative importance of the geostrophic and ageostrophic components. It was reported a high ageostrophic contribution to the kinetic energy at the eastern and northwestern basin, while the geostrophic component dominates the dynamics in the Alboran, the Algerian and the Ionian sub-basins. Here, based on the same formulation, LCS and relative dispersion from FSLE are computed for the geostrophic and ageostrophic velocity components using operational available products with the aim to investigate the transport and mixing properties of the different Mediterranean sub-basins.

This paper is organized as follows: Section 2 describes the data and contains a brief summary of the formulation used to obtain the velocity field. Section 3 exposes the applied methodology to 
analyze the case study. In Section 4, the acquired results in this report are reported and discussed. And, finally, Section 5 concludes the work with some highlighted points.

\section{Data}

\section{Velocity fields}

Sea surface currents are obtained following the methodology described in Morales-Márquez et al. (2020). We use a modified Ekman model for the surface currents, which includes the balance between Coriolis forces due to the mean and wave-induced motions and the surface wind and wave stress (Huang 1979; Polton et al. 2005). In order to find analytical solutions for the surface currents, that allows us using available wind and waves data from synoptic observations, we propose a simplified model, considering the steady state of the conservative wave-averaged Boussinesq horizontal momentum equation, within a uniform and steady surface gravity wave field (McWilliams and Fox-Kemper 2013) and in the presence of surface wind stress, and a small Rossby number $R=U / f L$. Following (Huang 1979; Polton et al. 2005; Morales-Márquez et al. 2020) these equations can be described using the complex notation (i.e, $\mathbf{U} \equiv u+i v$ and $\nabla=\frac{\partial}{\partial x}+i \frac{\partial}{\partial y}$ ) as:

$$
\mathrm{i} f\left(\mathbf{U}_{T}+\mathbf{U}_{s}\right)=-\frac{1}{\rho_{w}} \nabla P+\frac{1}{\rho_{w}} \frac{\partial \tau}{\partial z}-\mathbf{T}_{w d s}
$$

where $\rho_{w}$ is the water density, $\mathbf{U}_{\mathbf{s}}$ is the wave-induced Stokes drift, if $\mathbf{U}_{\mathbf{s}}$ the Coriolis-Stokes forcing, and where the horizontal mixing has been neglected and the vertical one is given by the stress $\tau$. With this simplification, we focus on the main current removing the small-scale Langmuir vortices given by the vortex-force term and neglecting non-linear advection terms. The momentum equations are split into a mean geostrophic and ageostrophic balances. Hence, the total velocity field, $\mathbf{U}_{\mathbf{T}}$, can be approximated as the sum of the geostrophic $\mathbf{U}_{g}$ related to the pressure term and the ageostrophic components resulting from wind and waves stress, $\mathbf{U}_{a}$. At this point, we remark that the Lagrangian velocity contribution $\left(\mathbf{U}_{s}\right)$ in the pressure gradient term is neglected since the geostrophic velocity is obtained from altimetry which averages all small contributions. The Stokes Coriolis force by itself is neither geostrophic nor ageostrophic. It might be considered as part of a "wavy geostrophic balance" including both Coriolis terms and the pressure gradient on the right hand side of eq. (1) (McWilliams and Fox-Kemper 2013; Suzuki and Fox-Kemper 
2016, e.g.), or alternatively as part of a "wavy Ekman balance" involving Stokes Coriolis, vertical mixing, the surface wind stress as a boundary condition, and momentum transfer due to waves [e.g.](McWilliams et al. 2014). In this paper, we take Stokes Coriolis and wave radiation and mixing effects to all be included in the latter collection of terms and thus as an ageostrophic contribution to the velocity. Under these assumptions and in the limit of an Ekman depth much larger than the Stokes layer depth $\left(h_{E k} \gg h_{S t}\right)$, as occurs in the Mediterranean Sea (Sayol et al. 2016), the effect of waves on the Eulerian currents can be modeled through a modification of the surface boundary condition (Polton et al. 2005; Wenegrat and McPhaden 2016). Using the Ekman-Stokes stress, $\tau=\rho A_{z}(z) \frac{\partial \mathrm{U}_{\mathrm{a}}}{\partial z}$, directly in the momentum equation, being $A_{z}$ the turbulent eddy viscosity, considered as a specific parameter, allows approximating the equation for the ageostrophic current as, (Huang 1979; Polton et al. 2005; Morales-Márquez et al. 2020):

$$
\text { if } \mathbf{U}_{a}=\frac{\partial}{\partial z}\left(\mathrm{~A}_{z} \frac{\partial \mathbf{U}_{a}}{\partial z}\right)-\mathrm{i} f \mathbf{U}_{\mathbf{s}}-\mathbf{T}_{w d s} .
$$

In this work, the momentum transfer from waves to the mean flow due to dissipation of wave energy $\left(\mathbf{T}_{w d s}\right)$ is neglected and the vertical viscosity profile $\left(\mathrm{A}_{z}\right)$ is assumed to be vertically uniform and only dependent on wind speed with the relation 1.2.10-4 $\mathbf{U}_{\mathbf{1 0}}^{2} \mathrm{~m}^{2} \mathrm{~s}^{-1}$ (Ekman 1905; Santiago-Mandujano and Firing 1990) for the whole basin. According to Polton et al. (2005) and Morales-Márquez et al. (2020), the momentum equation (Eq. (2)) can be solved as a two points boundary value problem with the modified Ekman-Stokes stress condition at the free surface and a vanishing condition at $z=-\infty$, obtaining the following analytical solution:

$$
\mathbf{U}_{a}(z)=\frac{\boldsymbol{\tau}_{w}}{\rho_{w} \mathrm{~A}_{z} m} e^{m z}+\frac{\frac{\partial \boldsymbol{S}}{\partial \boldsymbol{X}}}{\rho_{w} \mathrm{~A}_{z} m} e^{m z}+\frac{m^{2} \mathbf{U}_{s 0}}{4 k^{2}-m^{2}} e^{2 k z}-\frac{2 k m \mathbf{U}_{s 0}}{4 k^{2}-m^{2}} e^{m z},
$$

where $\tau_{w}$ is the wind stress, $\frac{\partial S}{\partial X}$ the radiation stress due to the waves at the sea surface, $k$ the wavelength, $\mathbf{U}_{s 0}=\mathbf{U}_{s(z=0)}$ and $m=\sqrt{\mathrm{i} f / \mathrm{A}_{z}}=(1+\mathrm{i}) \sqrt{f /\left(2 \mathrm{~A}_{z}\right)}$. The analytical solution with the Coriolis-Stokes forcing is shown to be in agreement with velocity profiles from reported observational data, improving the standard Ekman model (Polton et al. 2005). 
Following the same order of the components in Eq. (3), and depending on the physical forcing, the ageostrophic component can be split into:

$$
\mathbf{U}_{a}(z)=\mathbf{U}_{E}(z)+\mathbf{U}_{\tau_{s}}(z)+\mathbf{U}_{S}(z)+\mathbf{U}_{E S}(z)
$$

where $\mathbf{U}_{E}(z)$ represents the classical Ekman component, $\mathbf{U}_{\tau_{s}}(z)$ accounts for the surface current induced by the wave radiation stress, $\mathbf{U}_{S}(z)$ is the Stokes component, and $\mathbf{U}_{E S}(z)$ is the EkmanStokes component that accounts for the interaction between wind and waves acting in the entire Ekman layer (Polton et al. 2005). Here, $\mathbf{U}_{\mathbf{a}}$ is integrated over 1 meter depth since the mean Stokes layer depth is generally smaller than $2 \mathrm{~m}$ in the Mediterranean Sea (Sayol et al. 2016).

To obtain the ageostrophic component, we use waves and $10-\mathrm{m}$ height wind derived from the ERA-Interim reanalysis product, which uses a WAM wave model with the assimilation of available measurements ERS1 satellite wave height data (Janssen et al. 1997). These data are extracted from local GRIB code of the European Centre for Medium-Range Weather Forecasts (ECMWF). This reanalysis product has a temporal resolution of 6 hours from 1979 to 2019 and a spatial resolution of $1 / 8^{\circ}$ both in latitude and longitude over the Mediterranean Sea. A detailed description of these products can be found in Berrisford et al. (2011).

The geostrophic component is obtained from the equilibrium between the Coriolis force and the pressure gradients in the momentum equation for a steady, homogeneous and Boussinesq flow:

$$
u_{g}=-\frac{g}{f} \frac{\partial(\mathrm{SSH})}{\partial y}, \quad v_{g}=\frac{g}{f} \frac{\partial(\mathrm{SSH})}{\partial x}
$$

where SSH is the Sea Surface Height, $g$ the acceleration of gravity and $f$ the Coriolis parameter. In this work we use the absolute geostrophic velocity fields provided by Copernicus Marine Environment Monitoring Service (CMEMS) through the product Mediterranean Sea Gridded L4 $S S H$. These data have a daily temporal resolution, and are interpolated each 6 hours, in accordance with the ageostrophic velocities (see below), to compute the total velocity field in a regular mesh of $1 / 8^{\circ}$ over the entire Mediterranean Sea.

In recent years, some authors, such as McWilliams et al. (2015) and Wenegrat and McPhaden (2016), have included an additional ageostrophic component caused by the geostrophic stress in the Ekman model. This term is a large contributor to the ocean dynamics at low latitudes, while the 
Coriolis-Stokes stress has a higher influence at higher latitudes, as in the case of the Mediterranean Sea (Wenegrat and McPhaden 2016). Over the very surface layers of this basin, the geostrophic stress value is 5 times smaller than Coriolis-Stokes stress, involving less than 3\% in spring and summer and than $8 \%$ in winter and autumn of the wind stress strength (Figure 9 of Wenegrat and McPhaden 2016). In the light of the small geostrophic stress input on the surface dynamics at the Mediterranean Sea, the results of this study can be assumed immutable if this component is considered.

\section{Lagrangian drifter data}

We use data from a total of 690 (15 SVP (Surface Velocity Program) and 675 CODE (Coastal Ocean Dynamics Experiment)) surface drifters deployed between 1994 and 2005 by several institutions operating in the Mediterranean Sea and collected by the Italian National Institute of Oceanography and Experimental Geophysics (OGS) (Hansen and Poulain 1996; Menna et al. 2017).

The velocity fields obtained in the previous subsection, are validated with this drifters-database, resulting an averaged separation distance between real and virtual drifter trajectories smaller when the virtual trajectories are calculated with total velocity field (including wind and waves-induced currents) rather than only geostrophic field (Morales-Márquez et al. 2020).

\section{Dynamical regions of the Mediterranean Sea}

We evaluate the transport and mixing properties in the six dynamically homogeneous regions of the Mediterranean Sea (shown in Figure 1) reported in Morales-Márquez et al. (2020). These regions (henceforth SOM-regions: R1, R2, R3, R4, R5 and R6) are unveiled through a SelfOrganising Maps (SOM) analysis (machine-learning algorithm applied to an artificial neural network) based on the homogeneous contribution of the geostrophic and Ekman- and Stokes-induced currents to the total kinetic energy. The regions where the mesoscale ageostrophic kinetic energy is significant are identified especially in R1, while the regions where the geostrophic dominates the dynamics behavior are defined as R2 and R3. Being R4, R5 and R6 intermediate regions where, in some occasions, the ageostrophic component controls the surface ocean circulation. More details about this regionalization can be found in Morales-Márquez et al. (2020). 


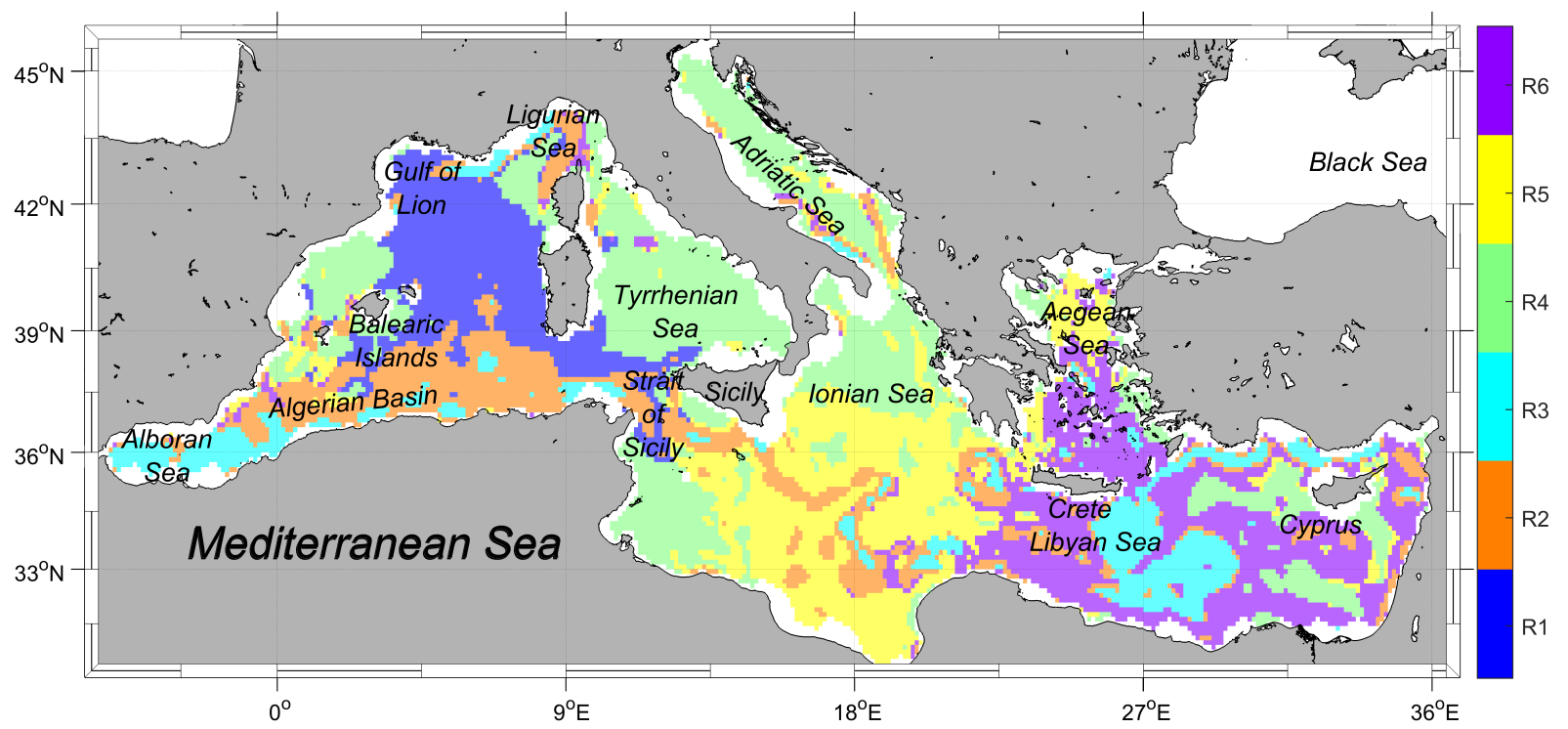

FIG. 1. Map of the Mediterranean Sea showing the main oceanographic features and the regions extracted through the SOM analysis applied to the total kinetic energy computed from the coupled geostrophic and ageostrophic (i.e. Ekman and Stokes induced currents) velocity fields. Figure adapted from Morales-Márquez et al. (2020).

\section{Lagrangian dynamics}

Neglecting diffusion effects, the trajectory of an infinitesimal and neutrally buoyant particle advected in a Lagrangian flow field $\mathbf{U}(\mathbf{r}, t)$ can be computed integrating the equation of motion

$$
\mathbf{r}(t)=\mathbf{U}(\mathbf{r}(t), t) .
$$

Here, we only consider two-dimensional fields, i.e. $\mathbf{r}=(x, y)$ with $x$ and $y$ the longitudinal and latitudinal coordinates, and where the ageostrophic velocity fields given by Eq. (3) are integrated in the vertical dimension, $\mathrm{z}$, over the first meter. The position of the particle between two consecutive times $t$ and $t+\Delta t$ is obtained integrating Eq. 6:

$$
\mathbf{r}(t+\Delta t)=\mathbf{r}(t)+\int_{t}^{t+\Delta t} \mathbf{U}(\mathbf{r}(t), t) d t
$$

Owing to the temporal and spatial discretization of the data sets an interpolation scheme has to be carried out to obtain the flow velocity $\mathbf{U}(\mathbf{r}(t), t)$ at the particle location (see Sayol et al. (2014); 
Van Sebille et al. (2018) for a comparison on numerical procedures). Trajectories given by Eq. (7) are integrated using a fourth-order Runge-Kutta scheme with a bilinear spatial interpolation of the velocity field and an integration time step of 1 hour, thus minimizing the numerical diffusion.

In order to analyze the influence of wind and waves on the total transport at the sea surface, the motion of the particles is computed using both the total and the geostrophic velocitiy fields:

$$
\frac{d \mathbf{r}_{T}(t)}{d t}=\mathbf{U}_{T}\left(\mathbf{r}_{T}(t), t\right)=\mathbf{U}_{g}\left(\mathbf{r}_{T}(t), t\right)+\mathbf{U}_{a}\left(\mathbf{r}_{T}(t), t\right),
$$

and

$$
\frac{d \mathbf{r}_{g}(t)}{d t}=\mathbf{U}_{g}\left(\mathbf{r}_{g}(t), t\right)
$$

\section{Relative dispersion statistics}

For the Lagrangian dynamical system defined in Eq. (6), a suitable metric to study the scaling properties of the relative dispersion is the averaged Finite-Size Lyapunov Exponent (FSLE) (Aurell et al. 1997), defined as:

$$
\lambda(\delta, \alpha)=\left\langle\frac{1}{\tau(\delta, \alpha \delta)}\right\rangle \ln \alpha,
$$

where $\delta$ is the initial separation between a pair of particles, $\alpha$ the amplification factor of separation and $\tau(\delta, \alpha \delta)$ the growth time of the separation distance between two particles from $\delta$ to $\alpha \delta$. The bracket $<>$ represents the average over a large number of realizations (pair of trajectories) for an initial separation $\delta$.

From the computation of FSLE over a wide range of initial separations $(\lambda(\delta) v s . \quad \delta)$ it can be inferred the contribution to relative dispersion by different length scales of motions. Information about the physical mechanism (e.g. diffusion, turbulence or chaotic advection) and the size of the flow structures that govern the Lagrangian dispersion processes, can be obtained from the dispersion regimes associated with the different scaling laws of FSLE (Artale et al. 1997; Aurell et al. 1997; Lacorata et al. 2001) identified by the exponent $\mu$ in the scaling $\lambda \sim C \delta^{\mu} . \quad \mu=0$ describes an exponential separation associated with non-local chaotic advection, that is, where larger structures than the analyzed separation scale between trajectories govern the dispersion (Boffetta et al. 2000); $\mu=-2 / 3$ corresponds to a Richardson dispersion scaling associated with a turbulent cascade (Richardson 1926); $\mu=-1$ indicates a ballistic dispersion regime associated with 
a constant shear flow, showing that the trajectories are located in different currents; and $\mu=-2$ represents a standard diffusion regimen associated with uncorrelated pair velocities, in which a particle trajectory independently separates from the other as a random walk (Taylor 1921).

To statistically assess the contribution of the different velocity components (geostrophic and ageostrophic) to the total relative dispersion, we compute $\lambda$ (Eq. (10)) and compare the results with the real drifters trajectories. The large number of uniformly distributed pairs of passive particle trajectories considered in the analysis, eliminates a possible bias due to the initial conditions (Artale et al. 1997). A total of 3098 pairs of particles are randomly released in each of regions identified in Morales-Márquez et al. (2020). The initial position of the particle \#1 for each of the pairs of particles is randomly selected inside the regions, as well as the time when the particle is launched throughout the time period of study. The position of the particle \#2 is chosen with a random angle with respect to particle \#1 and within a distance $5 \leq \delta \leq 300 \mathrm{~km}$. In order to analyze the sensitivity of the FSLE spectrum to the orientation of the sampling, we perform an experiment launching pair of drifters separated zonally and meriodinaly (Fig. 2).The values of the maximum Lyapunov exponents at small scales are significantly larger for meridional $\left(\sim 0.16\right.$ days $\left.^{-1}\right)$ than for longitudinal $\left(\sim 0.13\right.$ days $\left.^{-1}\right)$ initial separations Fig. 2 (red and blue lines respectively). Indeed, the scaling exponents obtained from the best fit of the FSLE curves at large scales shows a scaling exponent associated with a shear diffusion for meridional $(\mu=-1.21)$ and zonal $(\mu=-0.93)$ separation. Thus, sampling a wide range of directions minimizes possible anisotropic effects due to the direction of the initial separation vector. To resolve the relative dispersion associated with small coherent features and to avoid problems related to the time step of particle advection at small scales, the value of the amplification rate of separation $\alpha$ (Eq.(10)) must be smaller than 2 and not too close to 1. Here, we selected a fixed value of $\alpha$ as $\sqrt{2}$ (Lacorata et al. 2001; Haza et al. 2008). The analysis is also applied to the OGS drifters dataset described in section 2 with a drogue of 1 meter depth, considering only the pairs of drifters that are inside each region at least during 2 days. Since the Lagrangian model used to compute trajectories considers passive and infinitesimal particles, we neglected possible wind drag and other factors that modify the motion of the real drifter, assuming therefore trajectories of real drifting buoys as the best approximation of passive particle motion in the real ocean flow. 


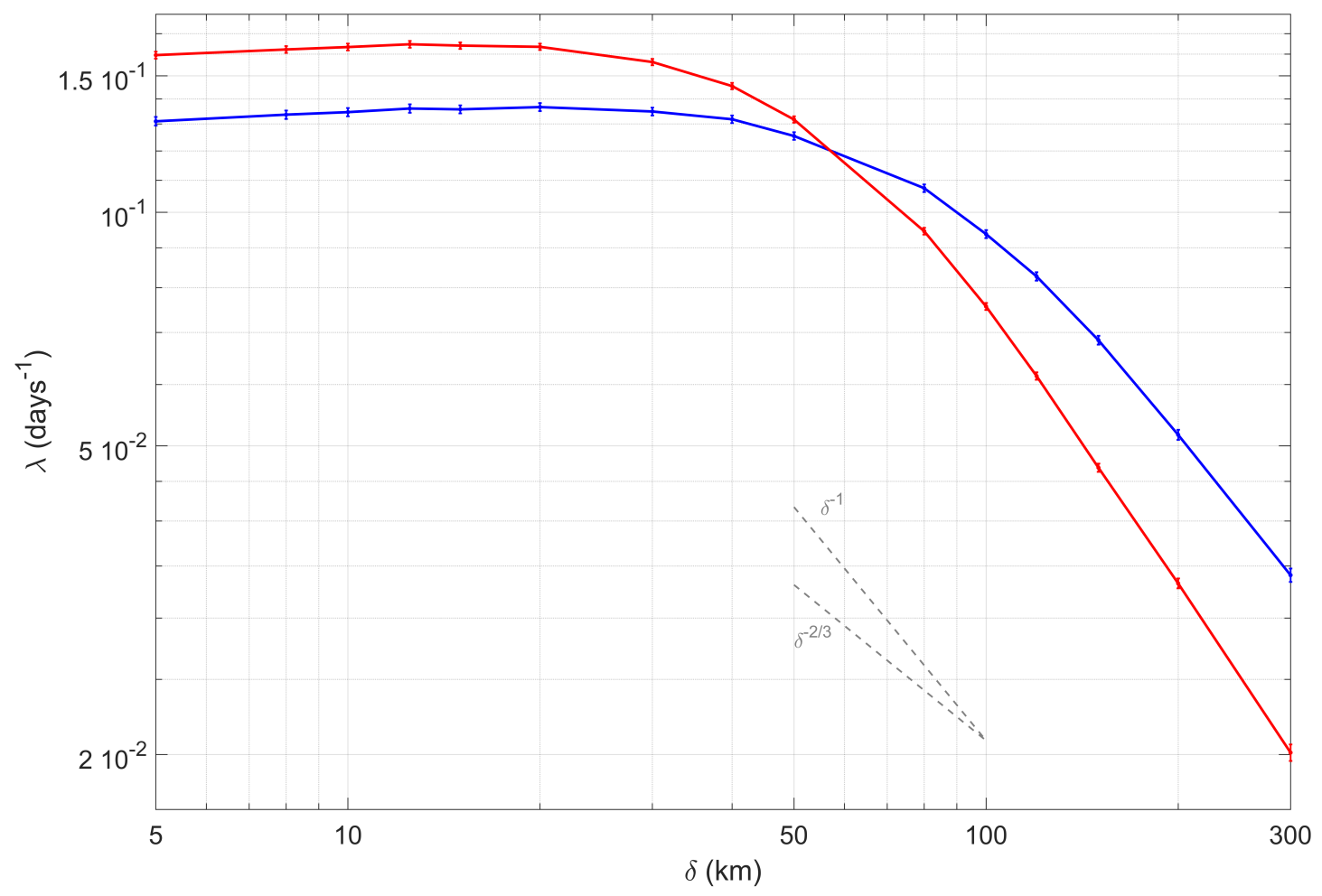

and

FIG. 2. FSLE spectrum, $\lambda(\delta)$ (in days ${ }^{-1}$ ) for different zonal (blue line) and meridional (red line) spatial scales $(\delta$, in $\mathrm{km})$ calculated with virtual drifters advected in the total velocity field $\mathrm{U}_{T}$ and without measuring the total final distance along an specific direction. The scaling exponents associated with ballistic/shear (-1) and Richardson (-2/3) dispersion regimes are included in the plot with dashed grey lines.

In a two-dimensional surface ocean flow, the characteristic scales in the Lagrangian dispersion can be analyzed independently for longitudinal and latitudinal directions splitting the FSLE into the zonal and meridional components as,

$$
\lambda_{x}\left(\delta_{x}, \alpha\right)=\left\langle\frac{1}{\tau\left(\delta_{x}, \alpha \delta_{x}\right)}\right\rangle \ln \alpha
$$

$$
\lambda_{y}\left(\delta_{y}, \alpha\right)=\left\langle\frac{1}{\tau\left(\delta_{y}, \alpha \delta_{y}\right)}\right\rangle \ln \alpha,
$$


being $\delta_{x}$ and $\delta_{y}$ the initial distances between pair of particles separated in the longitudinal or in the latitudinal direction. It should be noted that the final distance, in both definitions $\alpha \delta_{x}$ and $\alpha \delta_{y}$, is measured specifically along one direction: longitudinal and latitudinal, respectively.

The anisotropy in the dispersion process can be measured computing the difference between the zonal and meridional dispersion rates at a given scale $(\delta)$, through the Lagrangian anisotropy index (LAI) defined in Espa et al. (2014) as,

$$
L A I=\frac{\lambda_{x}\left(\delta_{x}\right)-\lambda_{y}\left(\delta_{y}\right)}{\lambda_{x}\left(\delta_{x}\right)+\lambda_{y}\left(\delta_{y}\right)},
$$

where $\delta_{x}=\delta_{y}=\delta$ and $\alpha$ have the same values to calculate $\lambda_{x}\left(\delta_{x}\right)$ and $\lambda_{y}\left(\delta_{y}\right)$. This dimensionless index varies between -1 and 1 , depending on whether the dispersion is dominated by latitudinal or longitudinal flows, respectively. Perfect isotropy is thus represented by zero value.

\section{Lagrangian Coherent Structures}

The FSLE technique can also be used to identify dynamical flow structures acting as barriers to transport of tracers (Boffetta et al. 2001; d'Ovidio et al. 2004; Hernández-Carrasco et al. 2011), the so-called Lagrangian Coherent Structures (LCS). In this case, we compute the minimum time that two fluid particles initially separated a distance $\delta_{0}$ need to be finally separated a distance $\delta_{f}$. At the position $\mathbf{r}$ and time $t$, the FSLE, is given by:

$$
\operatorname{FSLE}\left(\mathbf{r}, t, \delta_{0}, \delta_{f}\right)=|\tau|^{-1} \ln \frac{\delta_{f}}{\delta_{0}} .
$$

We remark that averages are not performed in this definition of the FSLE in order to have an explicit spatial-time dependence, in contrast with the original definition (Eq. 10), as well as, that $\alpha$ has to be large enough $\left(O\left(10^{1}\right)\right)$ to adequately distinguish regions of maximum stretching in the FSLE field. The largest Lyapunov values are organized along characteristic lines, namely LCS, which identify relevant oceanic structures like fronts, eddy boundaries and filaments. Since fluid particles can not cross them, such lines strongly constrain and organize the fluid motion around them, providing a kind of transport "template" (Shadden et al. 2005; Haller 2015). The minimum time $\tau$ is computed by integrating the trajectories of the four neighboring points of the analyzed one located at $\mathbf{r}$ and by selecting the associated particle that separates faster to a distance $\delta_{f}$. 
In this paper, LCS are computed from instantaneous FSLE maps using $\sim 3 \cdot 10^{6}$ pairs of backward trajectories initialized in a regular grid of $\delta_{0}=1 / 64^{\circ}$ over the entire Mediterranean Sea and with a final distance of $10 \delta_{0}$. Each daily FSLE map is computed using 44032 pairs of trajectories located in a regular grid with $1 / 8^{\circ}$ of spatial resolution, where the final fixed distance is $1^{\circ}$. The time averaged values in each grid point are computed only considering the days when the pair of particles trajectories do not reach the beach. In this way, there are some grid points where the time-average value is calculated with less amount of data, such as the points located near the coast of the Alboran Sea.

Similarly to LAI, we compute the Lagrangian Coherent Structure Anisotropy (LCSA), as:

$$
L C S A(\mathbf{r}, t, \delta, \alpha)=\frac{F S L E_{x}\left(\mathbf{r}, t, \delta_{x}, \alpha\right)-F S L E_{y}\left(\mathbf{r}, t, \delta_{y}, \alpha\right)}{F S L E_{x}\left(\mathbf{r}, t, \delta_{x}, \alpha\right)+F S L E_{y}\left(\mathbf{r}, t, \delta_{y}, \alpha\right)},
$$

where $\mathrm{FSLE}_{x}\left(\mathrm{FSLE}_{y}\right)$ are the Finite Size Lyapunov exponent obtained evaluating the pair separation along the longitudinal, $\delta_{x}$, (latitudinal, $\delta_{y}$ ) directions. This expression allows an assessment of the spatial variability of the effect of the flow anisotropy on the LCS. Depending on whether LCSA is positive or negative, the LCS is given by a higher contribution of the longitudinal or latitudinal separation of the trajectories, respectively. Note that to compute the LCSA we use large values of $\alpha(\gg 2)$ as used before for the LCS estimation.

\section{Results and discussion}

\section{Ageostrophic induced leakage across mesoscale LCS}

In this section, we analyze how ageostrophic Ekman and Stokes induced currents influence transport pathways in the upper ocean. We first compare the shape of the LCS derived from the total velocity field (henceforth $\left.\mathrm{LCS}_{T}\right)$ and from the geostrophic velocities $\left(\mathrm{LCS}_{g}\right)$ at a given time. Figures 3 a) and b) display an example of instantaneous maps of FSLE computed from total velocity $\left(\mathrm{FSLE}_{T}\right)$ and from the geostrophic velocity field $\left(\mathrm{FSLE}_{g}\right)$, respectively, for 19 January of 2005 at $12: 00$ UTM. FSLE $_{T}$ and FSLE $g$ values are in the same range, between 0 and 0.6 days $^{-1}$ (mixing time-scales of days/weeks) typical of mesoscale horizontal stirring (Hernández-Carrasco et al. 2012). A similar large scale pattern of intricate Lyapunov lines, associated with fronts and mesoscale eddy-like structures is exhibited, also when including the ageostrophic velocities. 

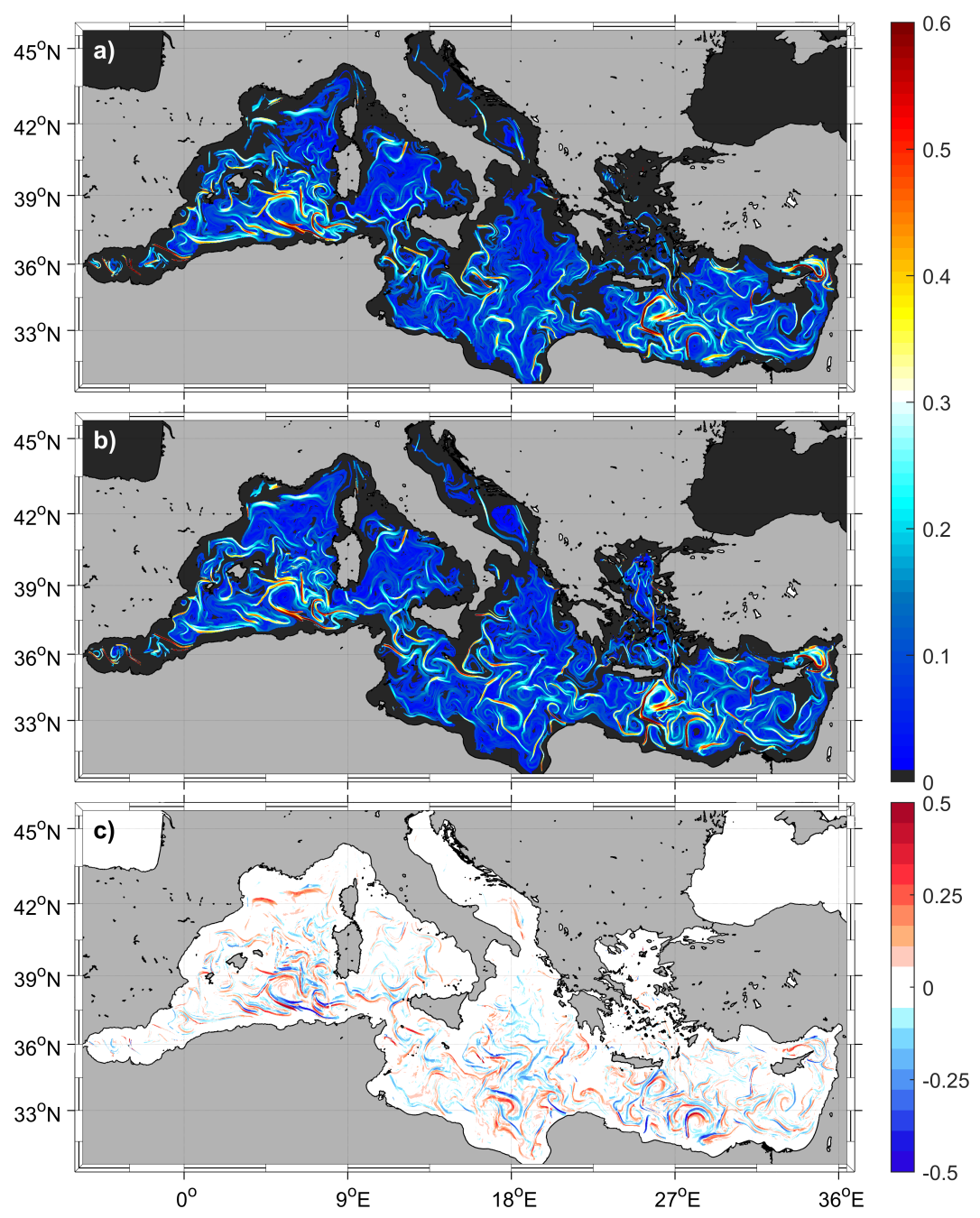

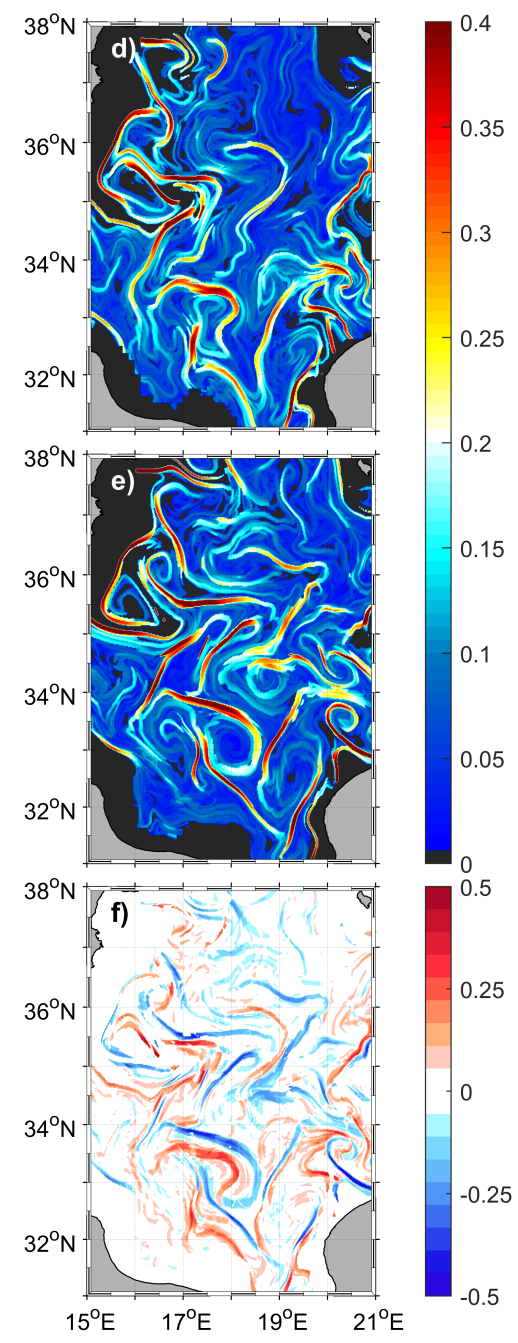

However, some discrepancies in the shape and intensity, as well as, in the position of $\operatorname{LCS}_{T}$ with respect to the $\mathrm{LCS}_{g}$, are clearly evident in Fig. 3, c) where we present the difference between

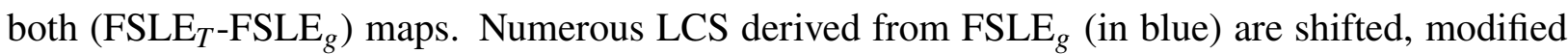
in intensity, or even totally dissipated when considering the total currents (in red).

FIG. 3. Spatial distribution of backward FSLE (days ${ }^{-1}$ ) in the Mediterranean Sea corresponding to January 19, 2005 at 12 : 00 UTM computed using 2791665 pairs of trajectories with a) total velocity fields, $\mathrm{U}_{T}$, and b) geostrophic velocity fields, $U_{g}$. c) Difference between total and geostrophic FSLE fields shown in a) and b) $\left(\mathrm{FSLE}_{T}-\mathrm{FSLE}_{g}\right)$. d), e) and f) are zooms in on the Ionian Sea of the $\mathrm{FSLE}_{T}, \mathrm{FSLE}_{g}$ and its difference, respectively. The initial separation is $\delta_{0}=1 / 64^{\circ}$ and the final separation, $\delta_{f}=10 \delta_{0}$. 
The impact of the ageostrophic currents on the LCS are evidenced if we zoom in on specific regions. Figures 3,d), e) and f) show large differences in the Lagrangian transport pattern obtained from both velocity fields. The shape of mesoscale vortexes and filaments are drastically altered in the $\mathrm{FSLE}_{T}$ map, as seen in the eddies over the Ionian Sea (e.g., the eddy centered at 18E - 33N), or even suppressed, as in the case of the filament-like LCS located over the northeast and central regions of the Ionian Sea. Additional examples showing that this ageostrophic modification of the LCS is not an isolated event are reported in the Appendix (Figure 4). This suggests that the ageostrophic currents could play an important role in the spreading of tracers in the ocean.
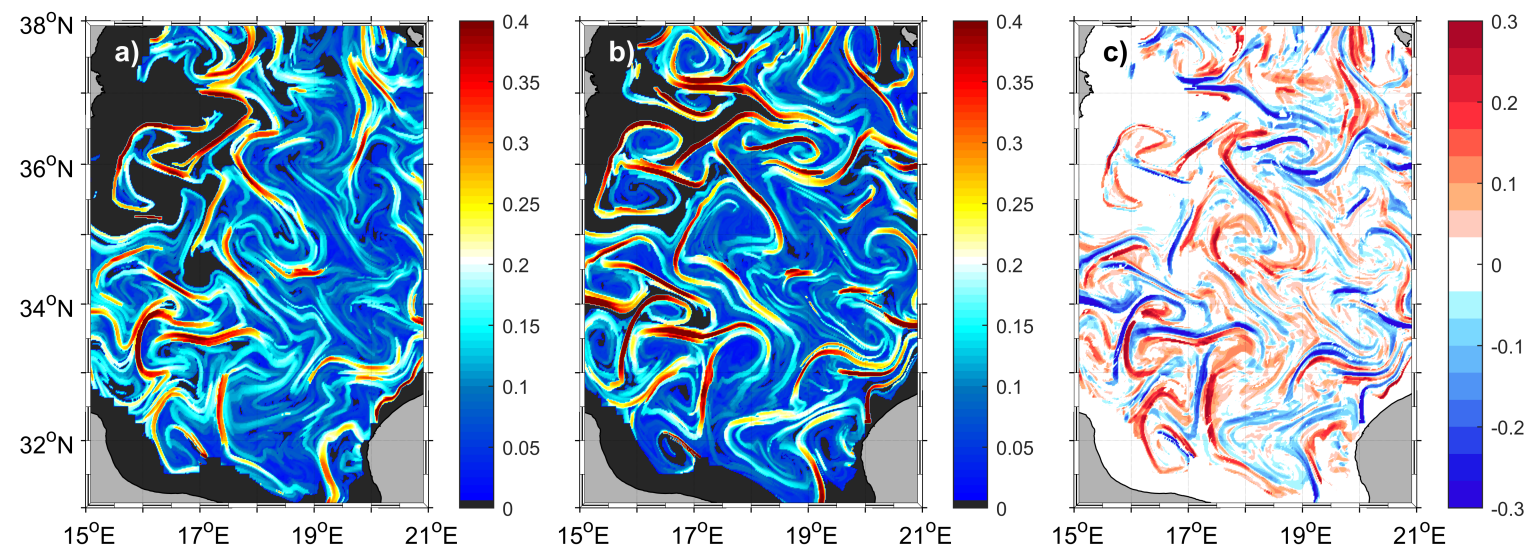

FIG. 4. Spatial distribution of backward FSLE $\left(\right.$ days $^{-1}$ ) in the Ionian Sea corresponding to February 5, 2014 at 6 : $00 \mathrm{UTM}$, computed from a) total velocity field, $\mathrm{U}_{T}$, and b) geostrophic velocity field, $\mathrm{U}_{g}$. c) Difference between maps a) and b). The initial separation is $\delta_{0}=1 / 64^{\circ}$ and the final separation, $\delta_{f}=10 \delta_{0}$.

To better illustrate the effect of the wind and waves induced currents on the Lagrangian distribution of transported material, we compare the evolution of a set of passive tracer trajectories advected in $\mathrm{U}_{T}$ with the same set of particles trajectories (released with the same initial conditions), advected in the geostrophic velocity field $\mathrm{U}_{g}$ (Figure 5). While tracers advected in the geostrophic field (cyan points) remain inside the mesoscale eddy, tracers inside total currents (pink points) leave the eddy, and eventually spread across the southwestern Mediterranean, toward the Alboran Sea. This suggests that wind and waves induced circulation could significantly impact on the permeability of the Lagrangian Coherent Structures (i.e. intense fronts and eddies) obtained from the geostrophic currents. The Ekman and Stokes developed motions permit a leak across the mesoscale transport barriers identified by the geostrophic LCS. It implies that, while the geostrophic transport 

barrier constrains strongly the motion of the particles inside the eddy when they are advected in the geostrophic flow, it becomes permeable when adding the ageostrophic component to the total currents. This fact could have profound consequences on the connectivity patterns, as well as, the retention capacity of eddies, which can be substantially influenced by the wind and waves conditions. There are obvious impacts of this result to dispersion and mitigation of pollutants and other flotsam, as barriers to transport are not detectable by SSH alone. 

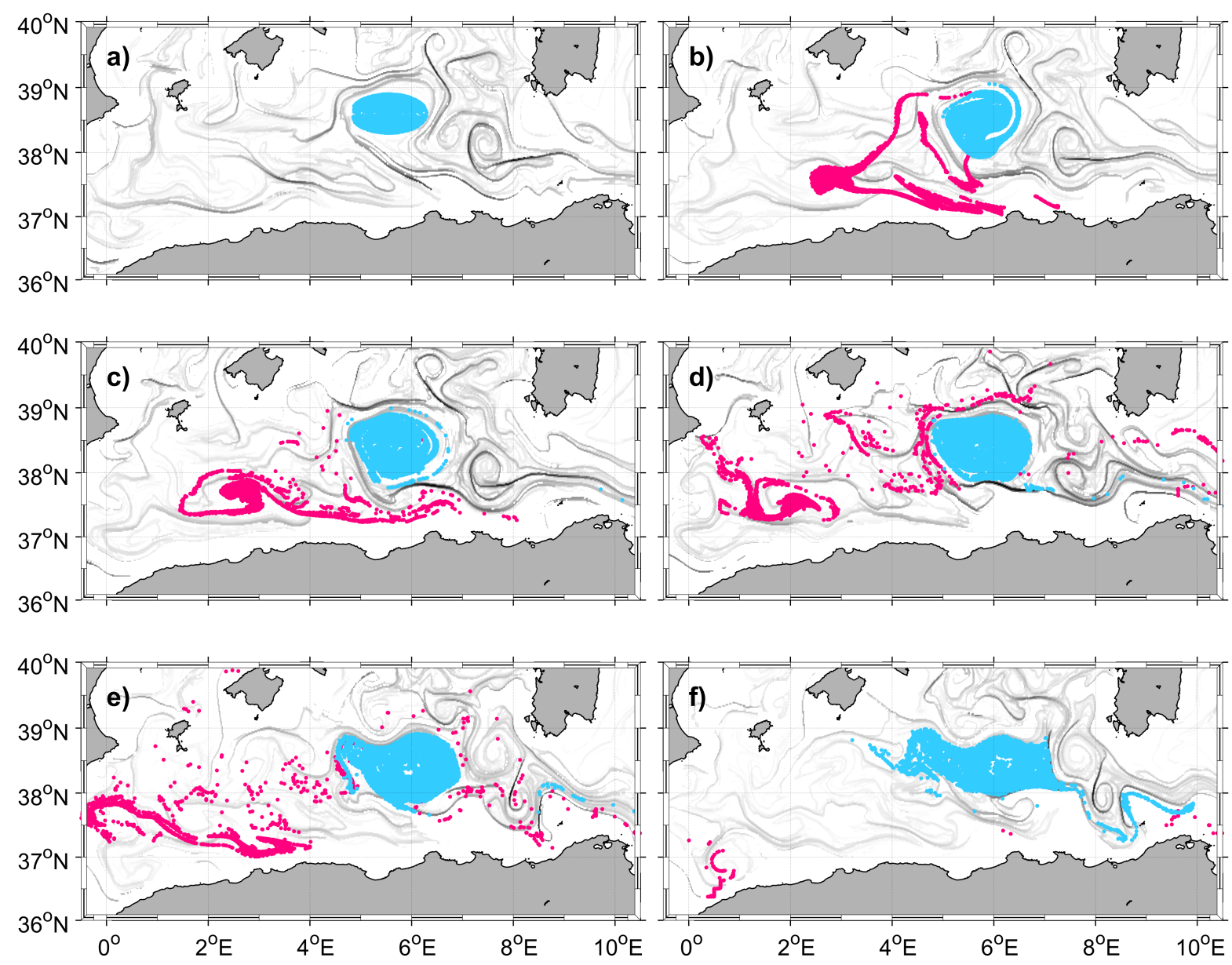

FIG. 5. Evolution during one month ( a) January 26, b) January 31, c) February 5, d) February 10, e) February 15 and f) February 20, 2005) of two sets of 10000 passive tracers launched with the same initial conditions in the interior of a mesoscale eddy. One set is advected by the geostrophic field (in cian) and the other set is advected by the total velocity field (in pink). The attracting geostrophic LCS are displayed in the background in gray (darker grey for more intense LCS). 


\section{Horizontal stirring variability}

We further analyze the low-frequency signature, large-scale signal, of the horizontal stirring by computing the time average of $\mathrm{FSLE}_{T}$ and $\mathrm{FSLE}_{g}$ over the 25-years of data. Areas with large values of averaged FSLE identify zones with more persistent horizontal stirring (d'Ovidio et al. 2004; Hernández-Carrasco et al. 2012). Fig. 6 shows that regions with more mesoscale activity are located in the Alboran Sea (associated with the Alboran gyres variability, with values of FSLE $_{T}$ around 0.6 days $^{-1}$ ) and the Gulf of Lion (associated with the Northern current), the Algerian basin (related to the instabilities of the Algerian Current) and the south of Crete in the eastern Mediterranean (associated with the variability of the intense gyres south of Crete, with values around 0.3 days $^{-1}$ ). The impact of the ageostrophic component on the horizontal stirring can be inferred by computing the difference between the time average of $\mathrm{FSLE}_{T}$ and $\mathrm{FSLE}_{g}$. The areas of more intense horizontal stirring due to ageostrophic mesoscale activity are characterized by large values of the relative difference between temporal averages of the total and the geostrophic FSLE with respect to the total FSLE, computed for each position (r) as:

$$
\% F S L E_{a}(\mathbf{r})=\frac{1}{T} \sum_{k=1}^{T} \frac{F S L E_{T}(\mathbf{r}, k)-F S L E_{g}(\mathbf{r}, k)}{F S L E_{T}(\mathbf{r}, k)} \cdot 100
$$

where $\mathrm{T}$ is the time period over which the time series are evaluated, being different in each position (r) depending on the simultaneous availability of both $\mathrm{FSLE}_{T}$ and $\mathrm{FSLE}_{g}$ fields (e.g. T= 8927 daily time steps corresponds to 24 years, if there exit values of $\mathrm{FSLE}_{T}$ and $\mathrm{FSLE}_{g}$ fields over the whole period of study at the same pixel). This allows knowing how is the ageostrophic contribution with respect to the total FSLE. Figure $6 \mathrm{~b}$ ) shows that areas where the mesoscale activity is increased by the effect of Ekman and Stokes (in red) are located in the Gulf of Lion and south of Crete. Regions where wind and waves have a suppressing effect on the geostrophic horizontal stirring (in blue) are observed in the western part of the Mediterranean Sea, near Sardinia and west Sicily, as well as, in the middle of the Eastern Mediterranean basin.

The average of FSLE is also calculated seasonally from 1994 to 2018 in order to characterize the regional impact of the intra-annual variability of the wind and waves conditions on the LCS. We only focus on the winter-summer differences (not shown all the seasons). The averaged FSLE $_{T}$ over winter months (December-January-February-March) is shown in Fig. 6, c) and over summer 

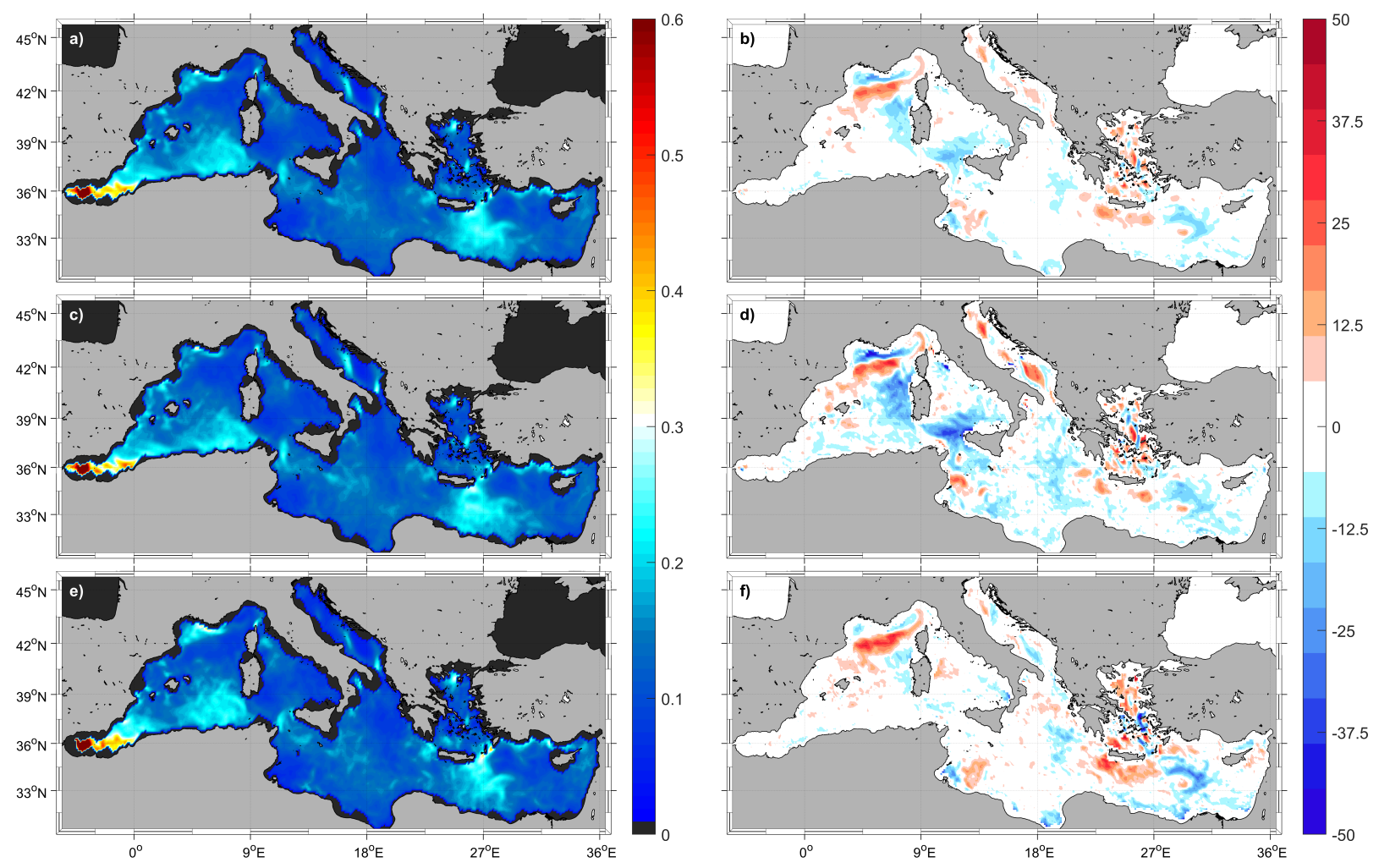

FIG. 6. Spatial distribution of the time average of backward $\mathrm{FSLE}_{T}$, in days ${ }^{-1}$, over: a) the 24 years of data (from 1994 to 2018); c) only averaging over winter months (DJFM); and e) only averaging over summer months (JJAS). Contribution of the ageostrophic currents proportional to the total horizontal stirring in \% (FSLE ${ }_{T}-$ $\left.\mathrm{FSLE}_{g}\right) / \mathrm{FSLE}_{T}$ ), for b) the total period; d) for winter; and f) for summer. The initial separation is $\delta=1 / 8^{\circ}$ and the final separation, $r \delta=1^{\circ}$.

(June-July-August) in Fig. 6, e). Clear differences between seasons are appreciated in the Gulf of Lion, Alboran Sea, Algerian basin and at the south of Crete, with higher mixing activity during summer. Note that in winter the mesoscale activity is almost cancelled in the Gulf of Lion. Similarly, we compute the normalized contribution of ageostrophic currents to horizontal mixing for winter (Fig. 6, d) and summer (Fig. 6, f). In winter, the ageostrophic component mainly exhibits an inhibitory effect of stirring, particularly significant in regions where the intense mistral and tramontane winds are developed in winter (Obermann et al. 2018; Soukissian et al. 2018), i.e. west Sicily and Sardinia, and the coastal region of the Gulf of Lion. Conversely, an increase of the mesoscale activity is observed over the south of Crete, the Adriatic and Aegean Sea, and in the south part of the Gulf of Lion. In summer, Ekman and Stokes currents substantially impact on 
the geostrophic horizontal stirring (with an increase up to $50 \%$ of $\mathrm{FSLE}_{T}$ ) around Crete and the Aegean Sea, likely caused by the persistent northerly etesian winds that prevail over the eastern Mediterranean during summer (Zecchetto and De Biasio 2007; Soukissian et al. 2018), and in the northwestern Mediterranean where, although characterized by low values of the total mesoscale activity, an increase is observed induced by the ageostrophic currents likely due to occasional intense mistral winds blowing during this season (Small et al. 2012).

A convenient quantity to characterize mixing activity in a specific region, is the spatial average of FSLE at a given time which allows to study the temporal variability over different regions. Here, we are interested in the variability of the horizontal mixing in the SOM-regions shown in Fig. 1. The time evolution of the spatial average of the $\mathrm{FSLE}_{T}$ and $\mathrm{FSLE}_{g}$ over the whole Mediterranean Sea is shown in Figure 7, a). Both $\mathrm{FSLE}_{T}$ and FSLE $g$ show a high temporal variability, with larger values of $\mathrm{FSLE}_{T}$ than $\mathrm{FSLE}_{g}$ most of the time, in particular during the 1997-1999 and 20122014 periods, and a global contribution of $6 \%$ of the total FSLE coming from the ageostrophic component. As expected (and in agreement with Fig. 6), we observe high stirring values (mean FSLE of $\sim 0.23$ days $^{-1}$ ) corresponding to regions characterized by the major mesoscale features, such as the persistent intense mesoscale eddies, i.e. Alboran and Crete gyres (R3), and jets, i.e. Algerian Current (R2). Intermediate mixing values correspond to northwestern and eastern basins (R1 and R6) while central basin (R5) and Adriatic and Tyrrhenian Sea (R4) display significantly lower values. While events of maximum ageostrophic contribution (up to $37 \%$ of the total FSLE in Fig. 6, d) occur in the northwestern regions (R1), the average contribution is larger $(\sim 9 \%)$ in the eastern basin (R6) and north-central basin, including the Adriatic, Tyrrenian and north Ionian seas (R4), and lower $(\sim 3 \%)$ in the Aegean Sea and the south Ionian Sea (R5). Intermediate mean contributions $(\sim 5 \%)$ are found in $\mathrm{R} 1, \mathrm{R} 2$ and $\mathrm{R} 3$. In contrast to the results obtained in Morales-Márquez et al. (2020), where it was reported a high ageostrophic impact on the total kinetic energy in R1, we obtain that wind and waves induced currents do not play an important role in the mixing activity in this region. This suggests that mesoscale variability generated by the ageostrophic component is more significant in the eastern basin and in the north and central part of the Mediterranean Sea than in the rest of the regions.

Another interesting feature depicted from the mean FSLE time series is the general positive trend experienced in the Mediterranean basin, suggesting a continuous increase of the global 
mixing activity. Trends of the horizontal stirring are computed based on a linear regression of the residual component of FSLE time series after it has been decomposed as a seasonal signal plus a residual component. The global linear trend in the Mediterranean basin for the 1994-2018 period is $1.27 \cdot 10^{-3}$ days $^{-1} /$ year for $\mathrm{FSLE}_{T}$ and $1.32 \cdot 10^{-3}$ days $^{-1} /$ year for $\mathrm{FSLE}_{g}$, which is equivalent to a mean mixing increase of $0.8 \%$ per year. Regional differences are evident. Higher positive trends marking the central basin of the Mediterranean, i.e. the south Ionian Sea and the Aegean Sea (R5) with values of $1.67 \cdot 10^{-3}$ days $^{-1}$ /year (equivalent to $1.2 \%$ per year), the Algerian basin (R2) with a value of $1.66 \cdot 10^{-3}$ days $^{-1} /$ year $\left(0.8 \%\right.$ per year) and the core of the major gyres (R3) with $1.63 \cdot 10^{-3}$ days $^{-1}$ /year $\left(0.7 \%\right.$ per year). This implies a substantial $\mathrm{FSLE}_{T}$ increase in these regions that can reach around 0.2 days $^{-1}$ in 100 years (twice the current mixing level). Slightly lower positive signal is found in the eastern basin (R6) with a value of $1.33 \cdot 10^{-3}$ days $^{-1} /$ year $(0.7 \%$ per year), and the lowest inter-annual variations are located in the northwestern Mediterranean and in the Tyrrhenian and Adriatic Sea (R1 and R4) with an increase of $(0.82$ and 0.78$) \cdot 10^{-3}$ days $^{-1} /$ year $(0.5$ and $0.4 \%$ per year). $\mathrm{FSLE}_{T}$ shows a trend slightly higher than $\mathrm{FSLE}_{g}$ in R1, R2 and R5, suggesting an increase of the mixing activity induced by the ageostrophic component in these regions. The obtained positive trends are globally less pronounced than those reported in Ser-Giacomi et al. (2020) obtained for future climate projections of mixing.

An additional feature that can be observed is a marked seasonal signal in the time series of both geostrophic and total mixing activity. Further information about this seasonal variability can be obtained by analyzing the mean climatology of FSLE using the 24 years of data. In general over the whole Mediterranean basin, the lower FSLE values are found in summer, being constant along the rest of the year, and with the larger difference between $\mathrm{FSLE}_{T}$ and $\mathrm{FSLE}_{g}$ in the autumn-winter period (Fig. 8 a), likely induced by the intense wave and wind conditions developed during autumn and winter. However, as evidenced in Fig. 8 (panels R1-R6), each SOM-region shows different seasonal behaviour. While minimum values are reached in summer in all the regions, maximum mixing are found in different months. Regions where the mixing activity is more intense, e.g. Alboran Sea (R3) and south Crete, exhibit the highest seasonal variability. The northwestern Mediterranean (R1), associated with the Northern Current, shows maximum values in the mixing activity during spring and autumn and minimum values during summer and winter. This fact is closely associated with the main wind climate of the Mediterranean Sea exposed in Soukissian et al. 
(2018), since even if the windiest season is winter, the ageostrophic component has the opposite direction of geostrophy in this region (R1), removing part of the mixing activity there. The most active currents, in terms of mixing, identified by R2 and R3 present only one maximum in spring and autumn, respectively, and practically constant values during the rest of the year. The eastern basin, mostly identified by R6, presents constant values except in summer. The north of the central basin and the Adriatic Sea (R4) present maximum values in autumn, while the south of the central basin and the Aegean Sea (R5) show two peaks during winter and spring. R2, R3 and R5 barely show a seasonal impact of the ageostrophic component in the total mixing. It is worth noting that while region R1 exhibits an important ageostrophic contribution to mixing in autumn and spring, in R4 and R6 this occurs in autumn and summer, coinciding with the presence of the persistent and intense regional winds (tramontane in R1 and etesian winds in R6).

\section{Dispersion properties}

We further analyze the effect of wind and waves induced current on the surface dispersion properties over different regions of the Mediterranean Sea. Following other authors (Corrado et al. 2017; Lacorata et al. 2019), we evaluate the dynamical importance of the ageostrophic currents in particle dispersion by computing the averaged FSLE $(\lambda)$ at different spatial scales using Eq. (10) (see Material and Methods section). Unlike Bouzaiene et al. (2020), we perform the average of $\lambda$ not over the conventional Mediterranean sub-basins but over the dynamically coherent SOMregions shown in Fig. 1. In Fig. 9, a), we show the FSLE analysis averaging only over the pair of particles launched during the same period when the pair of real drifters are available in each SOM-region, and being advected by the total velocity field (solid lines) and geostrophic velocity field (dashed lines). The FSLE curves show that the exponential separation rate $(\lambda(\delta) \sim$ constant) denoted by $\lambda_{M}$ (maximum mesoscale Lyapunov exponent) varies for the different regions (see Table 1). The largest $\lambda_{M}$ values are found for R2 and R3, regions characterized by the major Mediterranean mesoscale features: gyres, fronts and jets (Algerian current, Alboran gyres, etc.) with a value around $1.8 \cdot 10^{-1}$ days $^{-1}$, followed by R1 and R6 (regions experiencing a significant impact of wind stress) with $\lambda_{M} \sim 1.35 \cdot 10^{-1} \mathrm{days}^{-1}$, and the lowest $\lambda_{M}$ corresponding to R4 and R5 with $1 \cdot 10^{-1}$ days $^{-1}$. Similar values (same order of magnitude) were reported in Lacorata et al. 


\begin{tabular}{|c|c|c|c|c|c|c|c|c|}
\hline & & $\lambda-\mathrm{U}_{\mathrm{g}}$ & $\lambda-\mathrm{U}_{\mathrm{T}}$ & $\lambda_{x}-\mathrm{U}_{\mathrm{T}}$ & $\lambda_{y}-\mathrm{U}_{\mathrm{T}}$ & $\lambda$-Drifters & $\lambda_{x}$ - Drifters & $\lambda_{y}$ - Drifters \\
\hline \multirow{3}{*}{$\mathrm{R} 1$} & $\lambda_{M}$ & 1.30 & 1.36 & 1.22 & 1.11 & 7.96 & 18.33 & 14.21 \\
\hline & $\mu$ & -0.72 & -0.67 & -0.99 & -0.79 & -0.65 & -0.56 & -0.97 \\
\hline & $\delta_{M}$ & 40.48 & 35.88 & 80.01 & 50.54 & & & \\
\hline \multirow{3}{*}{$\mathrm{R} 2$} & $\lambda_{M}$ & 1.73 & 1.79 & 1.64 & 1.56 & 10.49 & 21.50 & 23.27 \\
\hline & $\mu$ & -0.72 & -0.74 & -0.67 & -0.91 & -0.77 & -0.82 & red-1.37 \\
\hline & $\delta_{M}$ & 61.48 & 61.73 & 72.32 & 60.03 & & & \\
\hline \multirow{3}{*}{$\mathrm{R} 3$} & $\lambda_{M}$ & 1.77 & 1.85 & 1.66 & 1.56 & 20.38 & 29.72 & 24.20 \\
\hline & $\mu$ & -0.96 & -0.66 & -0.43 & -0.87 & -1.47 & -1.26 & N/A \\
\hline & $\delta_{M}$ & 83.95 & 69.05 & 80.94 & 85.13 & & & \\
\hline \multirow{3}{*}{$\mathrm{R} 4$} & $\lambda_{M}$ & 1.00 & 1.03 & 0.82 & 0.74 & 6.91 & 15.94 & 12.34 \\
\hline & $\mu$ & -0.66 & -0.70 & -0.89 & -1.42 & -0.82 & -0.91 & -0.84 \\
\hline & $\delta_{M}$ & 38.79 & 41.06 & 73.28 & 80.69 & & & \\
\hline \multirow{3}{*}{ R5 } & $\lambda_{M}$ & 1.01 & 1.01 & 0.88 & 0.77 & 10.24 & 23.13 & 18.04 \\
\hline & $\mu$ & -0.97 & -0.92 & -0.75 & -0.63 & -0.99 & -1.02 & -1.03 \\
\hline & $\delta_{M}$ & 60.92 & 59.85 & 68.99 & 49.52 & & & \\
\hline \multirow{3}{*}{ R6 } & $\lambda_{M}$ & 1.29 & 1.30 & 1.28 & 0.99 & 7.72 & 22.50 & 32.95 \\
\hline & $\mu$ & -0.86 & -0.73 & -0.50 & -1.09 & -1.36 & -1.31 & -2.10 \\
\hline & $\delta_{M}$ & 57.92 & 54.03 & 45.05 & 66.83 & & & \\
\hline
\end{tabular}

TABLE 1. Values of $\lambda_{M}\left(\cdot 10^{-1}\right.$ days $\left.^{-1}\right), \delta_{M}$ (in $\mathrm{km}$ ) and the slopes $(\mu)$ resulting from the best fitting of the FSLE curves obtained using pairs of virtual drifters advected in $U_{g}$ (referred as to $\left.\lambda-U_{\mathrm{g}}\right), \mathrm{U}_{T}\left(\lambda-\mathrm{U}_{\mathrm{T}}\right)$ and pairs of real drifters ( $\lambda$-Drifters), and of their corresponding zonal and meridional component $\left(\lambda_{x}\right.$ and $\left.\lambda_{y}\right)$, computed for each SOM-region of the Mediterranean Sea. In all cases the obtained correlation coefficients $\left(\mathrm{R}^{2}\right)$ are larger than 0.95 except for the fit of $\lambda$-Drifters in $\mathrm{R} 1\left(\mathrm{R}^{2}=0.89\right)$ and $\lambda_{y}-\mathrm{U}_{T}$ and $\lambda_{x}$-Drifters in region $\mathrm{R} 2\left(\mathrm{R}^{2}=0.90\right.$ and 0.92 , respectively). Slopes associated with Richardson (shear) [standard] turbulent dispersion are indicated in bold black (in italic).

(2019), where $\lambda(\delta)$ was computed averaging for the whole Mediterranean Sea. The same ranking in the $\lambda$ values is observed using real drifters (see Fig. 9, b).

Comparing the FSLE curves obtained from geostrophic currents with the obtained for total currents, we observe that both $\lambda_{M}$ are rather similar over R5 and R6 and slightly higher as computed from the total velocities in regions R1, R2, R3 and R4. This suggests that wind and 
waves induced currents have more impact on the dispersion of tracers over these Mediterranean Sea regions, being less pronounced in the eastern sub-regions.

The spatial scale identifying the transition between the exponential and the power law separation rate, denoted as $\delta_{M}$, is different in each region. This scale could give some insight about the minimum size of the mesoscale structures governing the relative dispersion. In R1, $\delta_{M}$ is around $36 \mathrm{~km}$, followed by R4 $\delta_{M} \sim 41 \mathrm{~km}, \mathrm{R} 6 \sim 54 \mathrm{~km}, \mathrm{R} 5 \sim 60 \mathrm{~km}$ and R2 $\sim 62 \mathrm{~km}$, and finally in R3 $69 \mathrm{~km}$.

The best-fitting of the regional $\mathrm{FSLE}_{T}$ and $\mathrm{FSLE}_{g}$ curves $\left(\lambda-\mathrm{U}_{T}\right.$ and $\lambda-\mathrm{U}_{g}$, respectively) at larger scales return values of the slopes spanning from -0.97 to -0.66 (see Table 1). In all the regions the relative dispersion obtained from both $\mathrm{U}_{T}$ and $\mathrm{U}_{g}$ is associated with a Richardson's turbulent diffusion (scaling rate of $-2 / 3$ ), except for R3, R5 and R6 obtained from $\mathrm{U}_{g}$ and for R5 obtained from $\mathrm{U}_{T}$, where the scaling law is rather related to a ballistic or shear dispersion (scaling rate of -1 ). It means that in R1, R2 and R4 the main contributors to the separation rate at these large scales are structures with size comparable with the separation itself. Note that, in general, the obtained slope is slightly steeper for $\lambda$-U $\mathrm{U}_{g}$ than for $\lambda$-U $\mathrm{U}_{T}$, particularly larger over R3, where the regime dispersion at large scales moves from being associated with a Richardson turbulent dispersion in the total field to a shear dispersion in the geostrophic velocity field.

The relative dispersion for the real drifters is calculated selecting all the simultaneously available drifters in each SOM-region at least during 2 consecutive days. In Fig. A2 in the Appendix is shown the number of drifter pairs transects available for each scale and region. Similarly to the obtained for virtual drifters, R3 shows the higher value of $\lambda_{M}$, followed by R2 and R5; and finally R1, R6 and R4, although all the $\lambda$ curves converge at large scales. The FSLE spectrum in regions R2, R3 and R6 suggest a plateau between 5 and $20 \mathrm{~km}$ (Fig. 9, b), associated with a mesoscale exponential separation, and a ballistic/shear dispersion at scales larger than $20 \mathrm{~km}$, although this has been taken with caution due to the small number of pairs used in the average, particularly in R3 and R6 (Fig. A2 of Appendix). This FSLE plateau at small scales observed in regions R2 and $\mathrm{R} 3$ reflects the absence of relevant submesoscale features due to limited grid resolution and the dominance of the mesoscale structures in the dispersion, i.e. the major Mediterranean mesoscale eddies and the intense jets, such as the Algerian current and its propagation toward the Ionian sea. The other regions, R1 and R4 present a relative dispersion behavior associated with a Richardson 
scaling and, R5 associated with shear dispersion, in agreement with the results obtained in Lacorata et al. (2019) for the global Mediterranean analysis (see Table 1). Comparing these results with the obtained from virtual drifters, we observe that, as expected, the coupled geostrophic and EkmanStokes model underestimates relative dispersion at small scales (range $\sim\left[1^{\sim} 80\right]$ ) $\mathrm{km}$ as reported in Lacorata et al. (2019).

\section{Anisotropy of the Mediterranean Sea flow}

In this section, we study the anisotropy of the flow in the different regions of the Mediterranean Sea based on the analysis of the relative dispersion along orthogonal flow components. In particular, we compute the longitudinal and latitudinal FSLE given by Eqs. (11) and (12) where the initial and final separations of the trajectory pairs are evaluated exclusively along one of the orthogonal components. This allows to assess the contribution of the zonal and meridional separation rate to the total dispersion.

We start analyzing the scaling properties of the dispersion for total velocity field $\mathrm{U}_{T}$ in both directions. In Fig. 10, blue and red lines show the zonal and meridional FSLE spectrum $\left(\lambda_{x}\left(\delta_{x}\right)\right.$ and $\left.\lambda_{y}\left(\delta_{y}\right)\right)$, respectively, calculated with $\mathrm{U}_{T}$ for each SOM-region (R1-R6). Anisotropy of the flow is reflected in the different behavior of the zonal and meridional components of the relative dispersion. In all regions, values of $\lambda_{M}$ are larger for the zonal component than for the meridional (up to $20 \%$ greater). Values of $\lambda_{M}$ range between $(0.82-1.66) \cdot 10^{-1}$ days $^{-1}$ for the zonal FSLE curves and between $(0.74-1.56) \cdot 10^{-1}$ days $^{-1}$ for the meridional FSLE (see Table 1 and Fig. 10). Note that $\lambda_{M}$ values are larger when considering the total separation distance than only considering the separation along one of the orthogonal directions. This shows that while the leading expansion direction of the separation vector is not only aligned along one exclusive orthogonal direction but a combination of both. In general, the zonal component of the flow has a higher impact on the relative dispersion than the meridional, being more significant at larger scales. As a consequence, the spreading of tracers is more oriented along the zonal direction than along the meridional.

Regions R1, R2 and R5 show a $\delta_{M}$ significantly greater for the zonal than for the meridional FSLE, up to $30 \mathrm{~km}$ of difference in R1. This suggests that in these regions the coherent structures governing the zonal dispersion are larger than the meridional structures. In fact, these regions are dominated by intense and large currents flowing zonally, e.g., Northern Current, Algerian Current, 
etc. While the slopes obtained from the best-fitting of the $\lambda_{x}$ is closer to a Richardson dispersion type slope, $\lambda_{y}$ curves show a slope associated with shear dispersion, except for R1, where we find the opposite, and for R5 where both components follow the Richardson's law. This slight departure from the Richardson-like dispersion in the latitudinal FSLE suggests that particles are dispersed in this direction due to the effect of a latitudinal shear produced by separated currents along the latitude, e.g. in R2 the Algerian Current and its associated re-circulation sub-currents.

Next, we compute the LAI (eq. (13)) for each scale and region to further characterize the difference between the zonal and meridional FSLE and to identify the characteristic scales of the flow anisotropy. We report in Fig. 11 the scale dependence of LAI obtained for the FSLE of the total velocity field $\mathrm{U}_{T}$. While each region shows a different degree of anisotropy, over the small-scales range, in general LAI is constant with relatively small positive values, and over large-scales LAI increases as the separation distance grows. This confirms that at large scales the longitudinal flows have more impact on the dispersion processes than the latitudinal component, in particular in regions R1, R2, R4 and R6. Regions where the flow anisotropy is weaker are R3 and R5. To identify the threshold scales $\delta_{A}$ at which the presence of anisotropy becomes relevant in the dispersion processes we use the following criteria: we consider the spatial scale at which the LAI departures from the constant range values. We found different $\delta_{A}$ values depending on the region. The regions more affected by the Ekman and Stokes induced currents (R1 and R5) present small $\delta_{A}$ values $(\sim 15 \mathrm{~km}$ ), while the region $\mathrm{R} 6$, regions where geostrophic dynamics is dominant ( $\mathrm{R} 2$ and R3) and R4 are characterized with large values of $\delta_{A}(\sim 40,30,80$ and $120 \mathrm{~km}$, respectively). Fig. A1 of Appendix shows the LAI obtained for the available real drifters in each region. It should be noted that the scarcity of drifters trajectories produces a large statistical errors, practically over all the separation scales, and a robust characterization of the scale dependence of LAI can not be properly addressed (see Fig. A3 in the Appendix for more details about the number of available pair of real drifters used in these computations). The anisotropy from front-wave interactions predicted in Suzuki and Fox-Kemper (2016) — sharper fronts in the down-Stokes direction and weaker fronts in other directions - is not expected to be estimated by this method, as the Stokes-front coupling using the 2D flow variables is not sufficient to drive frontogenesis. 

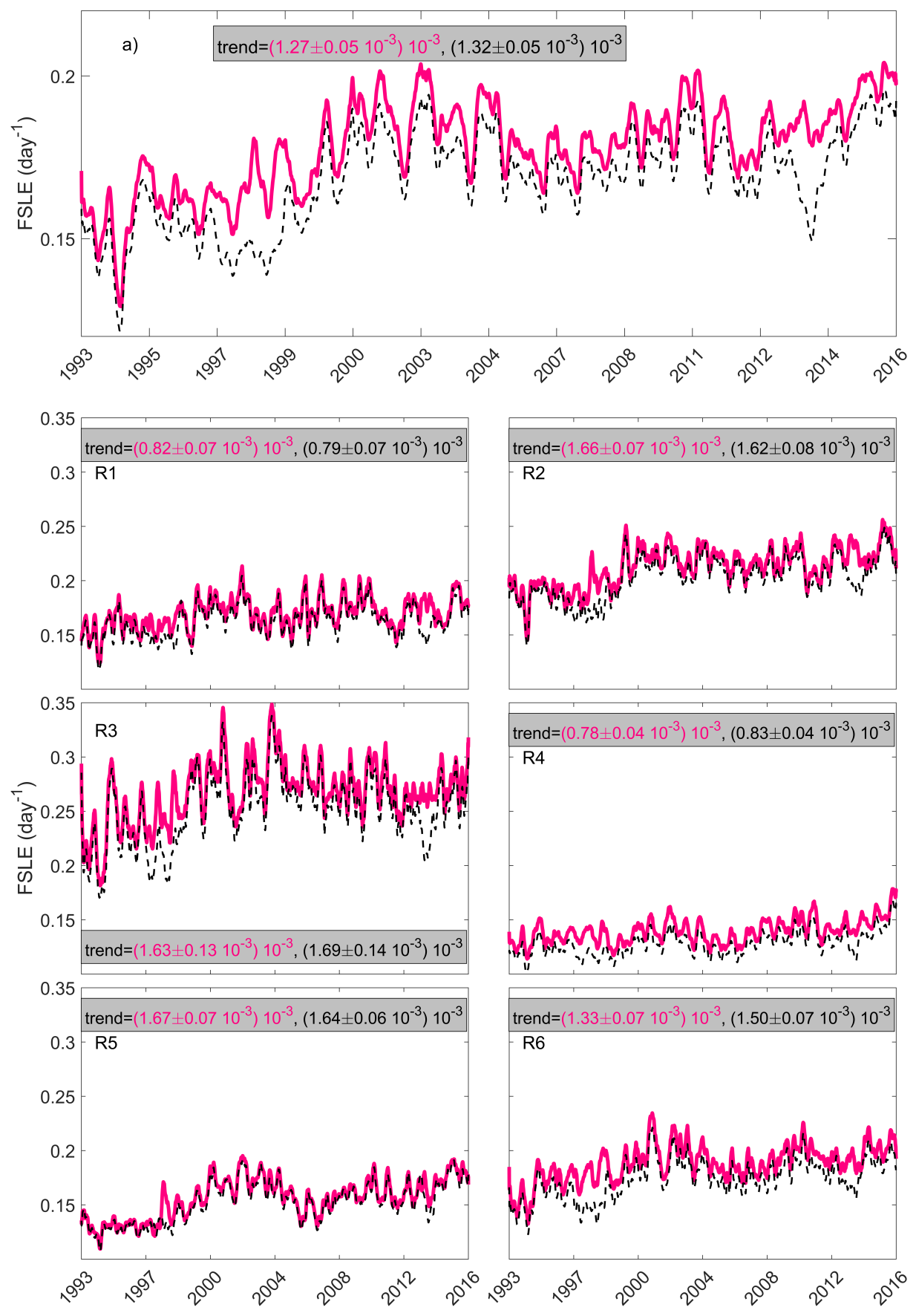

FIG. 7. Time evolution, from 1994 to 2018, of the spatial average of daily FSLE $_{T}$ (bold pink lines) and FSLE $g$ (dashed black lines), in days ${ }^{-1}$, over the whole Mediterranean Sea (panel a); and over the SOM-regions (panels R1-R6) shown in Fig. 1. The linear trends of the FSLE time series, expressed in days ${ }^{-1} / y e a r$, are included in each plot. Only trends with a significance $p \leq 0.01$ are included. FSLE is computed using $\delta_{0}=1 / 8^{\circ}$ and $\delta_{f}=$ $1^{\circ}$. 

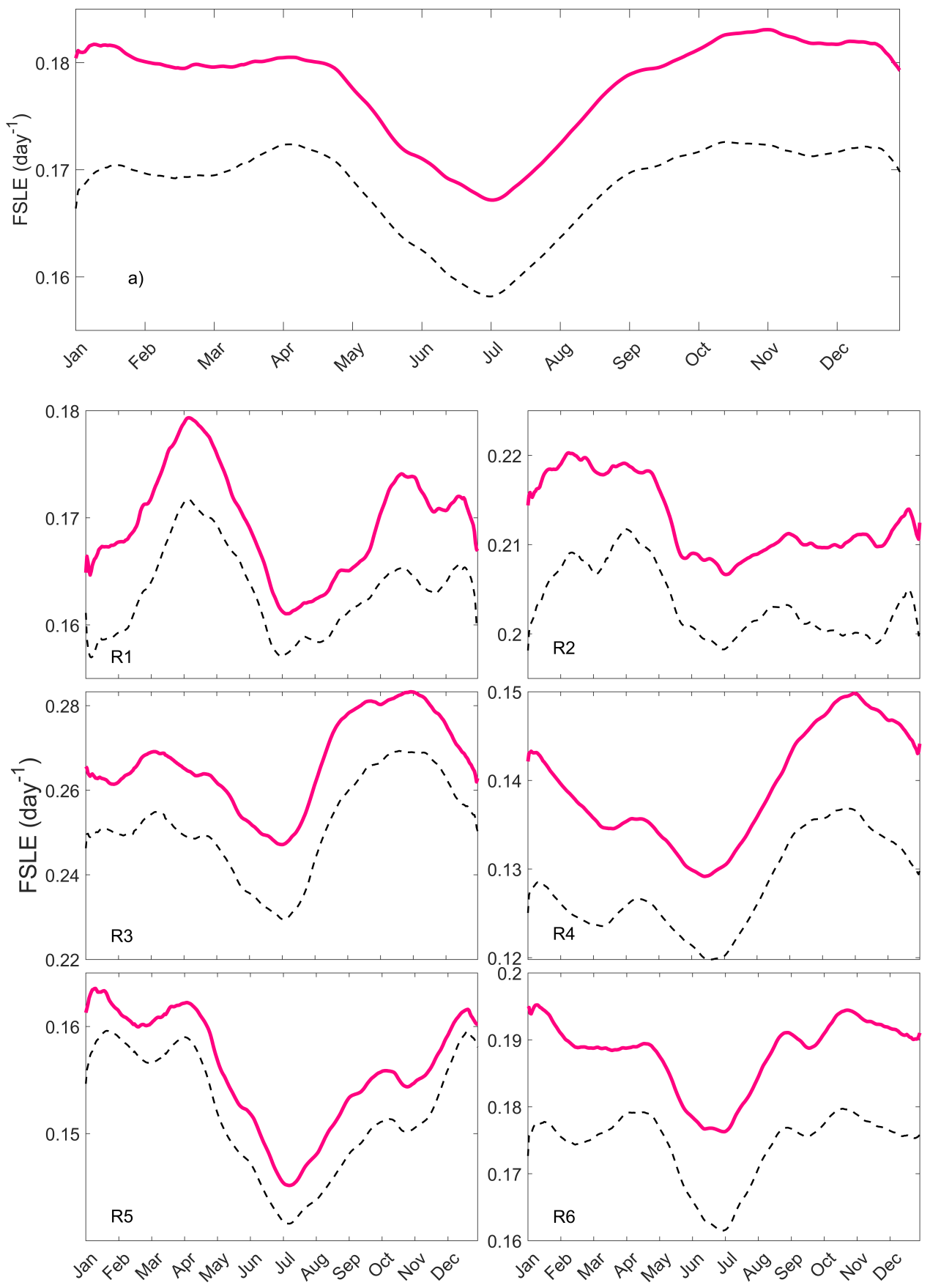

FIG. 8. Mean annual cycle of daily FSLE in days ${ }^{-1}$ (climatological daily mean over 24 years of data): a) for the whole Mediterranean Sea; R1-R6) for the SOM-region (bold pink lines correspond to $\mathrm{FSLE}_{T}$, and black-dashed lines to $\mathrm{FSLE}_{g}$ ). FSLE is computed using the $\delta_{0}=1 / 8^{\circ}$ and $\delta_{f}=1^{\circ}$. 

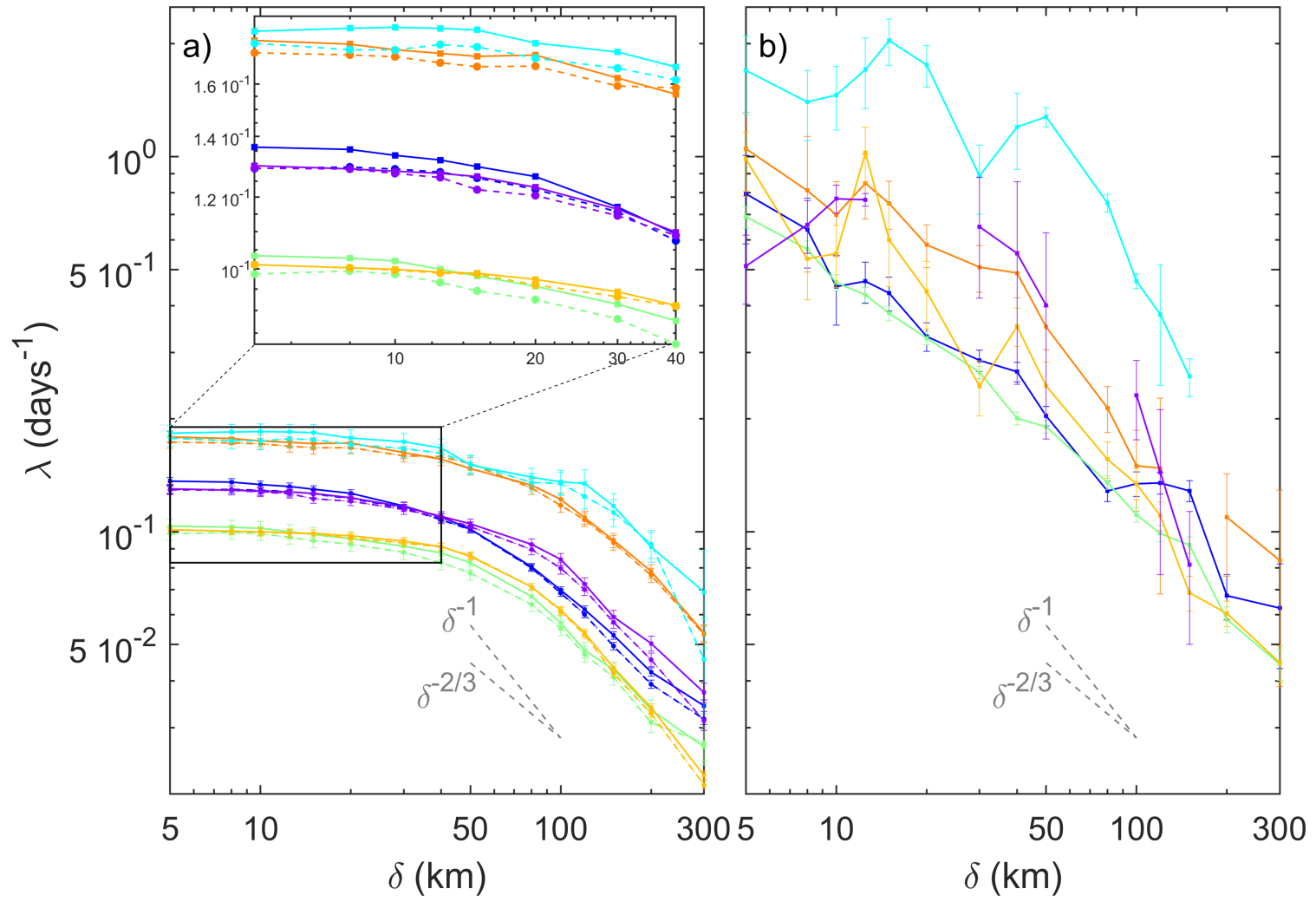

FIG. 9. FSLE curves $(\lambda(\delta))$, in days ${ }^{-1}$, at different spatial scales, in $\mathrm{km},(\delta)$ calculated with a) virtual drifters advected in the total velocity field (solid line) and in the geostrophic field (dashed line); b) with the real drifters. Each color corresponds to the averaged FSLE value over all the pairs of virtual drifters homogeneously launched in the SOM-regions identified in Fig. 1, and deployed at the same time period of the available pairs of real drifters in the corresponding SOM-region. The scaling exponents associated with ballistic/shear (-1) and Richardson $(-2 / 3)$ dispersion regimes are included in the plot with dashed grey lines. 

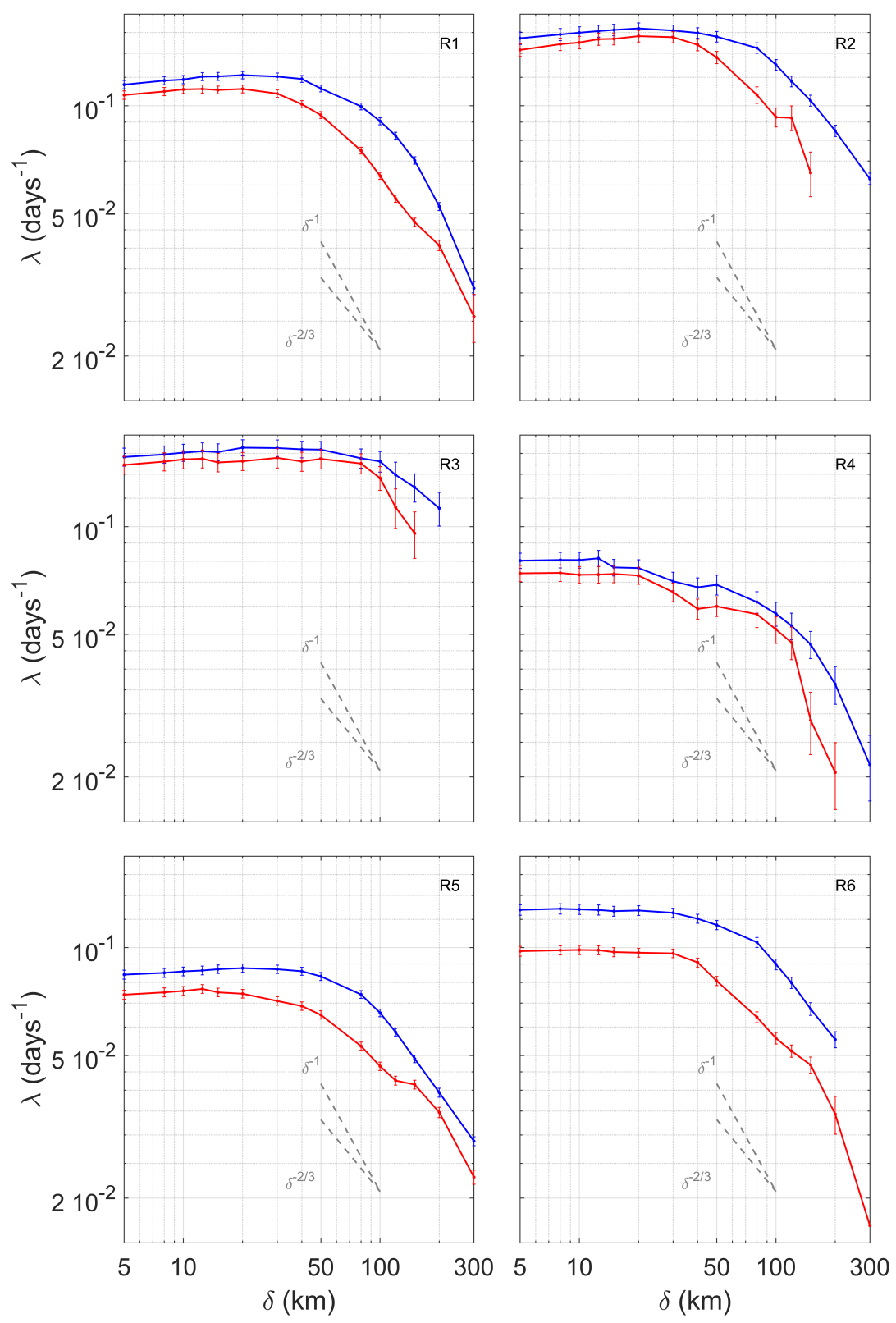

FIG. 10. Zonal (blue lines) and meridional (red lines) FSLE curves (in days ${ }^{-1}$ ) given by $\lambda_{x}\left(\delta_{x}\right)$ and $\lambda_{y}\left(\delta_{y}\right)$, respectively (see Eqs. 11 and 12), at different spatial scales ( $\delta_{x}$ and $\delta_{y}$, in $\left.\mathrm{km}\right)$ calculated for pairs of virtual drifters advected in the total velocity field $U_{T}$. Each subplot (R1-R6) corresponds to the averaged FSLE values over all the pairs of virtual drifters launched in the SOM-regions identified in Fig. 1, and deployed at the same time period of the available pairs of real drifters in the corresponding SOM-region. The scaling exponents associated with ballistic/shear (-1) and Richardson (-2/3) dispersion regimes are included in the plot with dashed grey lines. 


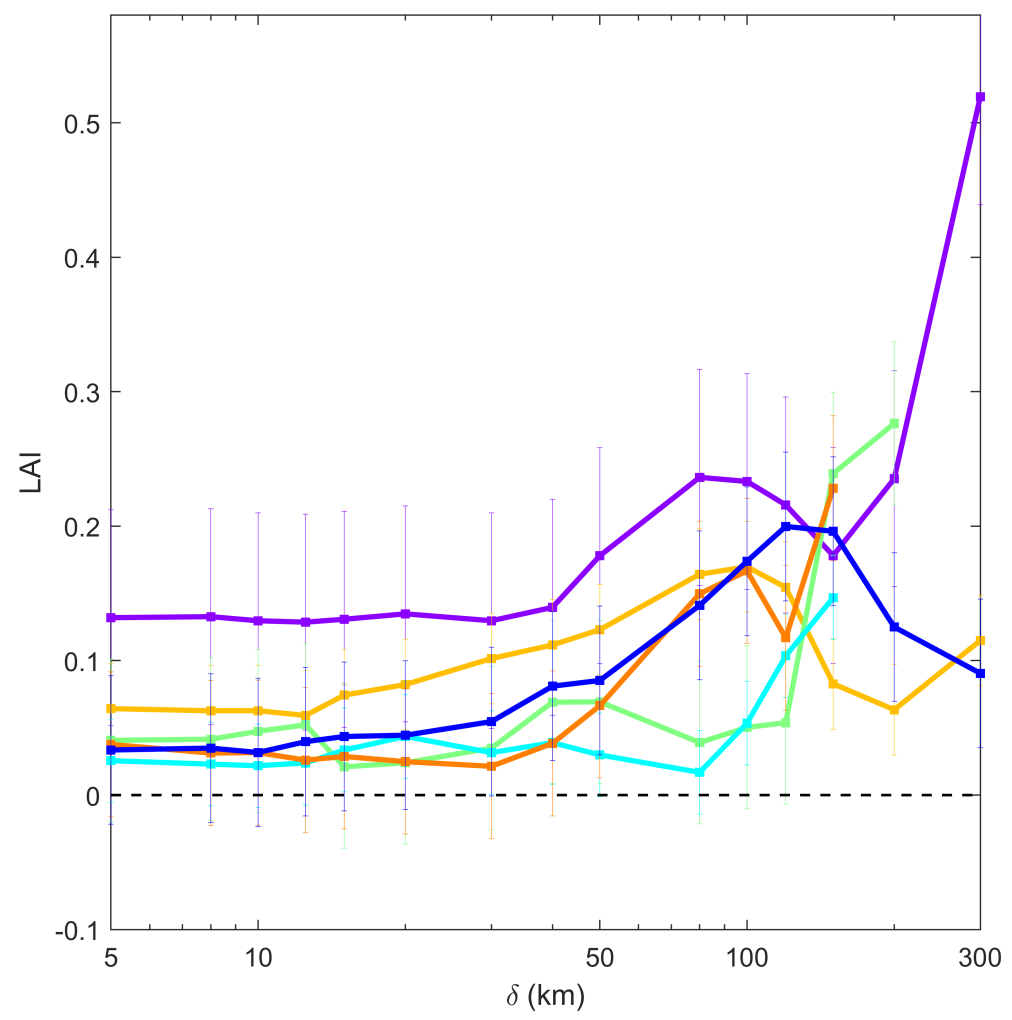

FIG. 11. Scale dependence of LAI computed with virtual drifters advected in the total velocity field for each SOM-region of the Mediterranean Sea. Colors correspond to SOM-regions identified in Fig. 1. The dashed black line represent the isotropy (LAI=0). 
We finally study the effect of the flow anisotropy on the Lagrangian Coherent Structures given by LCSA, and on the mixing activity obtained from $\angle \mathrm{LCSA}\rangle_{T}$, where the brackets represent a temporal average over the time period $\mathrm{T}$. To focus the analysis on the typical size of the mesoscale structures, LCSA is computed for final separation scales of $1^{\circ}$. In Fig. 12, a) the spatial distribution of $\langle\mathrm{LCSA}\rangle_{T}$ averaged over the 24 years of $\mathrm{U}_{T}$ data is depicted. Positive values (in red) indicates that the mixing activity is dominated by the longitudinal FSLE and negative $<$ LCSA $>_{T}$ (in blue), by the latitudinal FSLE. In general, we find that, across the Mediterranean basin, the zonal LCS are more significant than the meridional, in particular in the Eastern basin and the Adriatic Sea where $<$ LCSA $>_{T}$ reaches values up to 0.5 . This suggests that the zonal flow plays an important role in the mixing activity in Mediterranean Sea. The meridional FSLE dominates in specific regions, such as the northwestern basin and the south of Sicily, with the highest negative $<$ LCSA $>_{T}$ located along the Northern current, with values around -0.5. This implies that while in the eastern basin, the intense LCS (and thus transport barriers) are meridionally oriented (and the fluid flow is thus stretched with more intensity along the meridional direction), in the northwestern basin the LCS are zonally oriented. In the central basin (Ionian Sea) the flow is more isotropic. To identify regions showing seasonal variability of the anisotropy, we compute the average of the LCSA over the winter months (Fig. 12, c) and over the summer period (Fig. 12, e) across the 24 years of data. Slight seasonal differences are found in specific locations, such as along the Adriatic Sea and the Balearic Sea with an intensification of the longitudinal mesoscale mixing, while it is weakened at the surroundings of Create in summer, because this is time when etesian wind is stronger there. 

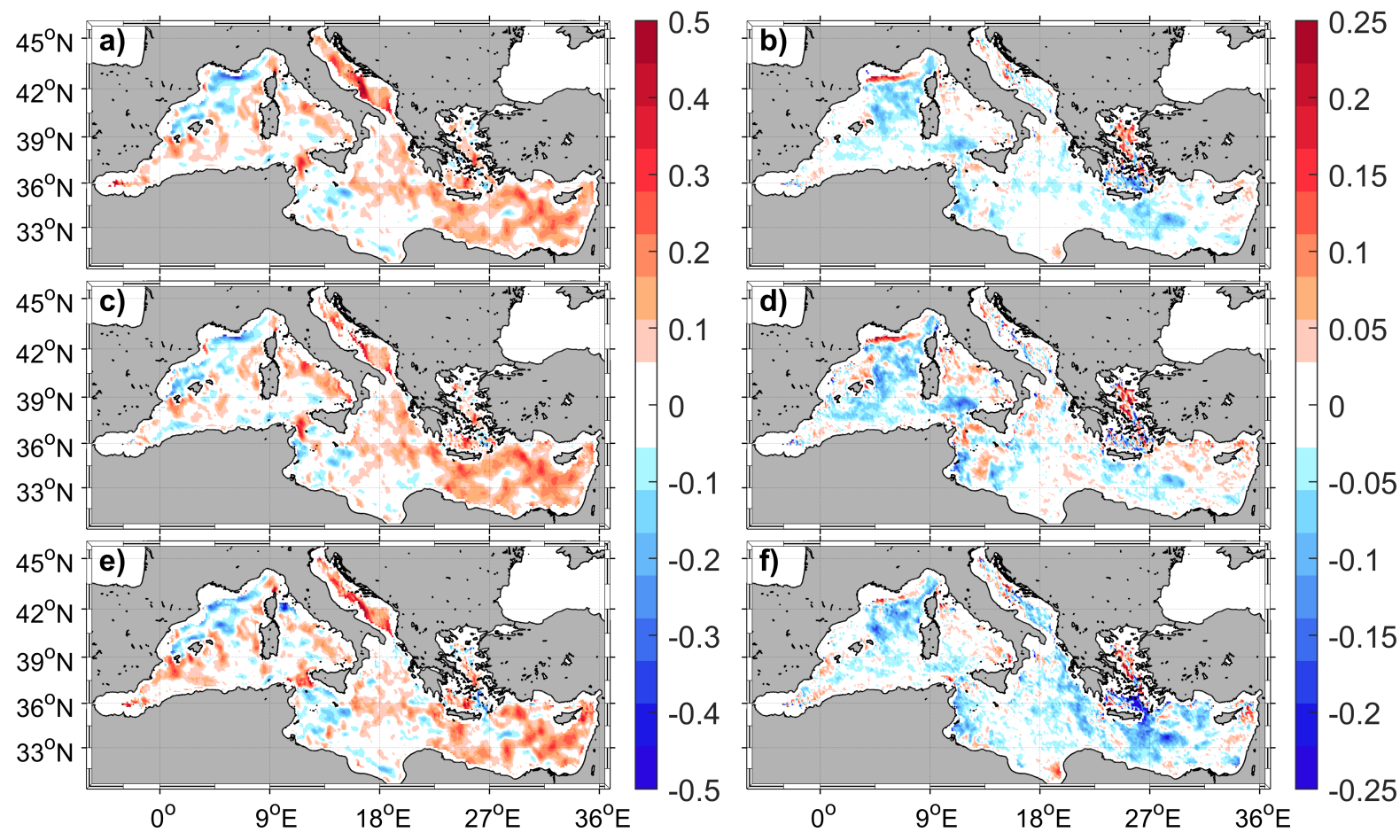

FIG. 12. Maps of the time average of LCSA computed over the 24 years of data (from 1994 to 2018); for the a)

$\mathrm{U}_{T}, \mathrm{~b}$ ) the difference between the LCSA obtained for $\mathrm{U}_{T}$ and for $\mathrm{U}_{g} . \mathrm{c}$ ) and d) are the same as $\mathrm{a}$ ) and b) but only averaged over winter; and e) and f) the same as a) and b) but only averaged over summer. The initial resolution is $\delta=1 / 8^{\circ}$ and the final resolution, $r \delta=1^{\circ}$. 
In order to further study the anisotropy of the flow associated with ageostrophic component, we compute the difference of the $<\mathrm{LCSA}>$ obtained for $\mathrm{U}_{T}$ with the $<\mathrm{LCSA}>$ for $\mathrm{U}_{g}$. The spatial distribution of the 24 years average of this difference is plotted in Fig. 12, b). We observe that while the ageostrophic FSLE are rather isotropic $(\operatorname{LCSA}=0)$ in the Ionian Sea, the Algerian basin and the most easterly part, significant negative $<$ LCSA $>$ values are located over the northwestern basin, north of Sicily and south of Crete. In these regions, the wind and waves main direction is North-South, which explains the ageostrophic contribution to the latitudinal mixing (Zecchetto and De Biasio 2007; Obermann et al. 2018). Positive ageostrophic contribution to <LCSA $>$ values are concentrated along the Northern current and the Aegean Sea, suggesting that Ekman currents and Stokes drift induces a zonal flow which has a large impact on the mixing properties in these regions. Furthermore, this ageostrophic meridional increase (zonal increase) of mixing is more intense during winter over the Strait of Sicily (Norther current) (see Fig. 12, d), and more intense at the south of Crete in summer (Fig. 12, f). This seasonal variability is in agreement with the seasonal intensification of the corresponding regional winds (Zecchetto and De Biasio 2007; Obermann et al. 2018).

\section{Conclusions}

With this work, we have analyzed the horizontal mixing and transport properties at the upper layer of the Mediterranean Sea associated with the wind and waves generated fluid particle motions. We have combined data from real drifters trajectories and the output of a Ekman modified model applied to 24 years of satellite altimetry observations and winds and waves derived from the ERAinterim reanalysis data. Although we have used data from drifters, in the present work we are not interested in reproducing the submesoscale dispersion, but large-scale features of the flow.

We have found that the ageostrophic component not only can drastically modify the mesoscale LCS, but also the direction of the tracer spreading and the retention capacity of geostrophic eddies. Consequently, this fact makes us also question about the classical view of the role of some oceanographic features, such as geostrophic transport barriers identified through LCS, in governing the spreading of particles or even in controlling the connectivity of the flow across different oceanic regions. 
FSLE strongly vary depending on the region. The main hot spots of horizontal mixing in the Mediterranean Sea are associated with the major Mediterranean mesoscale features. The horizontal mixing is not very intense in the majority of the Mediterranean basin, but it concentrates at some specific locations, such as inside the main mesoscale gyres (e.g. in the Alboran and Crete gyres) and other mesoscale features active enough to produce high stirring as the topographic generated eddies originated from the interaction of boundary geostrophic flows (e.g. the Algerian and Northern boundary currents) with steep topographic slopes. Wind and wave induced mixing is significant in the northwestern basin and the south of Crete with a contribution up to $37 \%$, but also showing a suppressing effect of mixing activity in the north part of the Algerian basin and Sicily.

As depicted from our results, the sub-regions unveiled from the SOM analysis of the total kinetic energy exhibit a different annual cycle of the horizontal mixing, as measured by averaged FSLE. While all regions show a minimum values in summer time, maximum mixing activity occurs in different months depending on the region. The strongest seasonal variability are identified in the northwestern basin and the Ionian and Aegean Seas. Regions characterized by high intensity of horizontal mixing, i.e. Alboran Sea, Algerian and Eastern basin, also present the lowest seasonal variability, due to the presence of quasi-permanent mesoscale features. The wind and waves induced mixing is also reflected in the seasonal variation of the ageostrophic contribution to the FSLE, clearly appreciated in the western basin and in the Aegean Sea during the periods of mistral, tramontane and etesian winds intensification.

We have found important inter-annual variations (positive trends) in the mixing activity, that can reach values up to $1.07 \%$ year $^{-} 1$ in regions such as the Alboran, Algerian basin and the south of the Ionian Sea. Otherwise, northwestern basin, the Tyrrenian, Adriatic and the north of the Ionian Sea experience a lower, $0.5 \%$, mixing interannual increase. The global linear trend of $\mathrm{FSLE}_{g}$ is higher than $\mathrm{FSLE}_{T}$, suggesting a decline of mixing induced by wind and wave currents. FSLE trends could be explained by the relatively larger interannual intensification of the eddy amplitude and higher variability, relative to a smaller contribution from the wind and wave stress. Note that the intensity of geostrophic velocities are associated with larger SSH gradients of eddies with a large increase in amplitude. The FSLE trend could have significant consequences in the transport of essential oceanic variables, such as heat, carbon, etc., with climatic implications. Consequently, 
determining changes to the FSLE field is fundamental to our understanding of the Mediterranean Sea and its potential response to climate change. Further studies should be performed in order to unravel the physical mechanism and forcings leading this mixing activity variation, for instance analyzing correlations with the regional wind stress and their long-term variability.

The Lagrangian dispersion in each region has been characterized from virtual and real pairs of trajectories. The scale-dependence property of $\lambda$ allowed us to estimate the maximum dispersion value and the scaling exponents of the pair dispersion spectrum, useful to determine the physical processes controlling the dispersion. The provided information was used to infer the typical scales of the flow structures governing the dispersion in each region. The obtained results from the synthetic trajectories show an exponential regimen at small-scales associated with chaotic advection in all the regions (as expected because of the coarser resolution of the gridded data), followed by a Richardson like dispersion regime (consistent with a 2D inverse cascade) at large scale also in all the Mediterranean, except for the south of Ionian and the Aegean Sea, which are characterized by a shear turbulent diffusion due to separation of particles by uncorrelated currents. Regions with the higher dispersion level are those dominated by the major mesoscale features, in agreement with the regions of high mixing activity. The same regional pair dispersion hierarchy is found for the real drifter trajectories confirming the results obtained from the virtual trajectories. Additionally, we have found similar dispersion regimens, except for the region dominated by the major mesoscale features where the low number of drifters produces a large standard error in the fitting of the slope.

The anisotropy analysis of the relative dispersion reveals the existence of higher contribution of the zonal flow to the dispersion properties in the Mediterranean basin except for the surroundings of Gulf of Lion and Sicily, which are characterized by a higher meridional dispersion. As shown in the results the mesoscale coherent structures are larger along the zonal direction than in the meridional. The temporal averaged mixing anisotropy in the Mediterranean Sea is broadly longitudinal (positive LCSA values), revealing that the zonal flow dominates the mesoscale mixing activity. However, there is a central region that can be considered isotropic regarding mixing properties. During winter seasons, the anisotropy at the western basin is intensified, while for summer periods is higher in the eastern; being crucial the ageostrophic component contribution. In general, the ageostrophic 
component induces an increase of the meridional mixing, likely due to the north-south wind and wave intensification. These transport properties have profound consequences on the regional distribution of quantities of biological or physical interest, providing novel insights on the distribution of drifting organisms, ${ }_{724}$ pollutants and, more generally, any tracer that is transported by the flow. 
Acknowledgments. The authors acknowledge financial support from Ministerio de Ciencia e Innovación (MCI), Agencia Estatal de Investigación (AEI) and Fondo Europeo de Desarrollo Regional (FEDER) through MOCCA Project (RTI2018-093941-B-C31). V. Morales-Márquez is supported by a postdoctoral contract from the University of Toulon - Mediterranean Institute of Oceanography (MIO). In addition, this work was performed while V. Morales-Márquez was supported by a FPI grant from the Ministerio de Ciencia, Innovación y Universidades. I. Hernandez-Carrasco acknowledges the Vicenç Mut contract funded by the Government of the Balearic Island. This work was partially performed while A. Orfila was a visiting scientist at the Earth, Environmental and Planetary Sciences Department at Brown University through a Ministerio de Ciencia, Innovación y Universidades fellowship (PRX18/00218). B. Fox-Kemper was supported by ONR N00014-171-2963. Authors would also like to thank Guglielmo Lacorata for the valuable discussions.

Data availability statement. All data are accessible from https://apps.ecmwf.int/ datasets/data/interim-full-daily/levtype=sfc/ and from https://resources. marine. copernicus.eu/?option=com_csw\&view=details\&product_id=SEALEVEL_MED_ PHY_L4_REP_OBSERVATIONS_008_051. Drifters dataset is a product provided by OGS on personal request (Menna et al. 2017). 


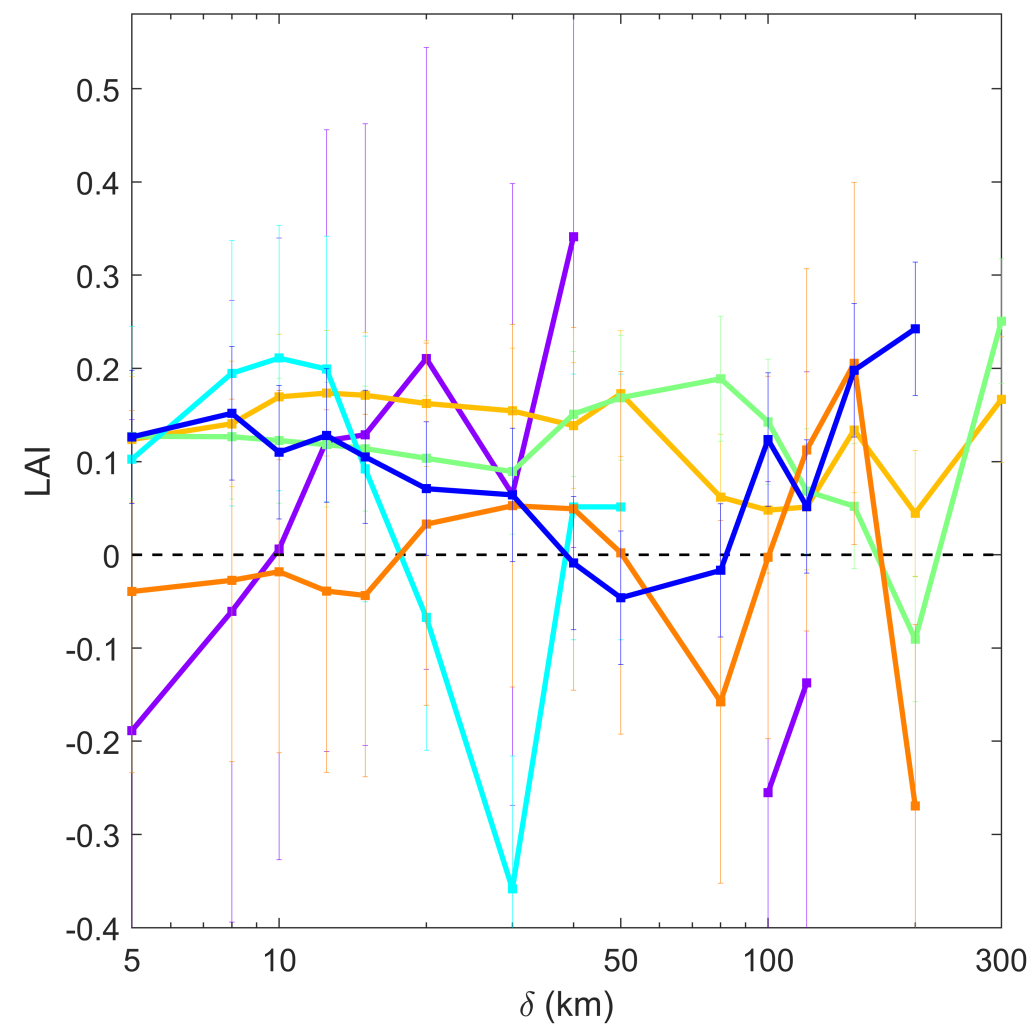

FIG. A1. Scale dependence of LAI computed with drifters for each SOM-region of the Mediterranean Sea.

Colors correspond to SOM-regions identified in Fig. 1. The dashed black line represent the isotropy (LAI=0). 

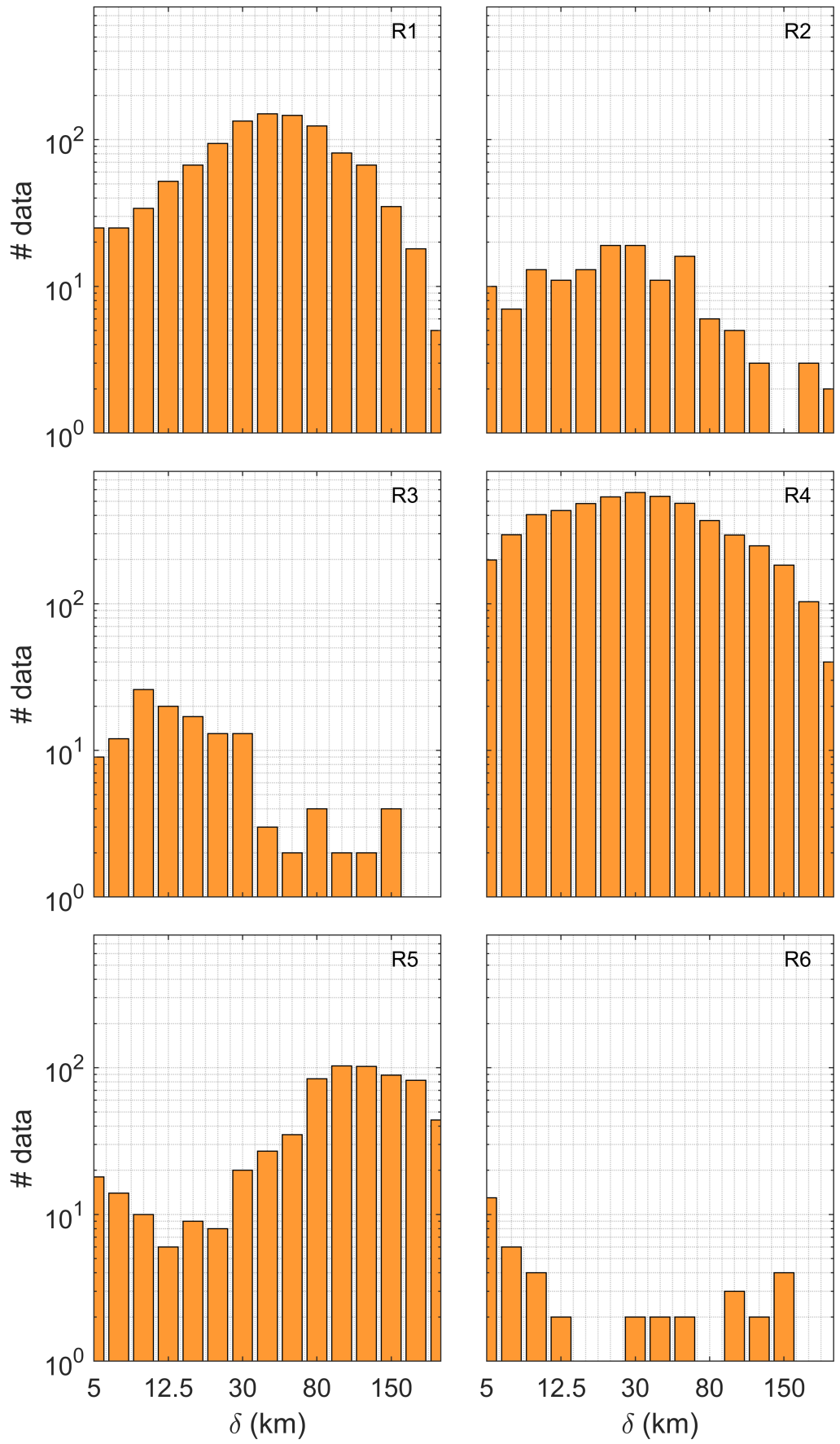

FIG. A2. Number of tracks of pairs of real drifters that are inside each region at least during 2 days used in the regional dispersion analysis shown in Fig. 9, b. 

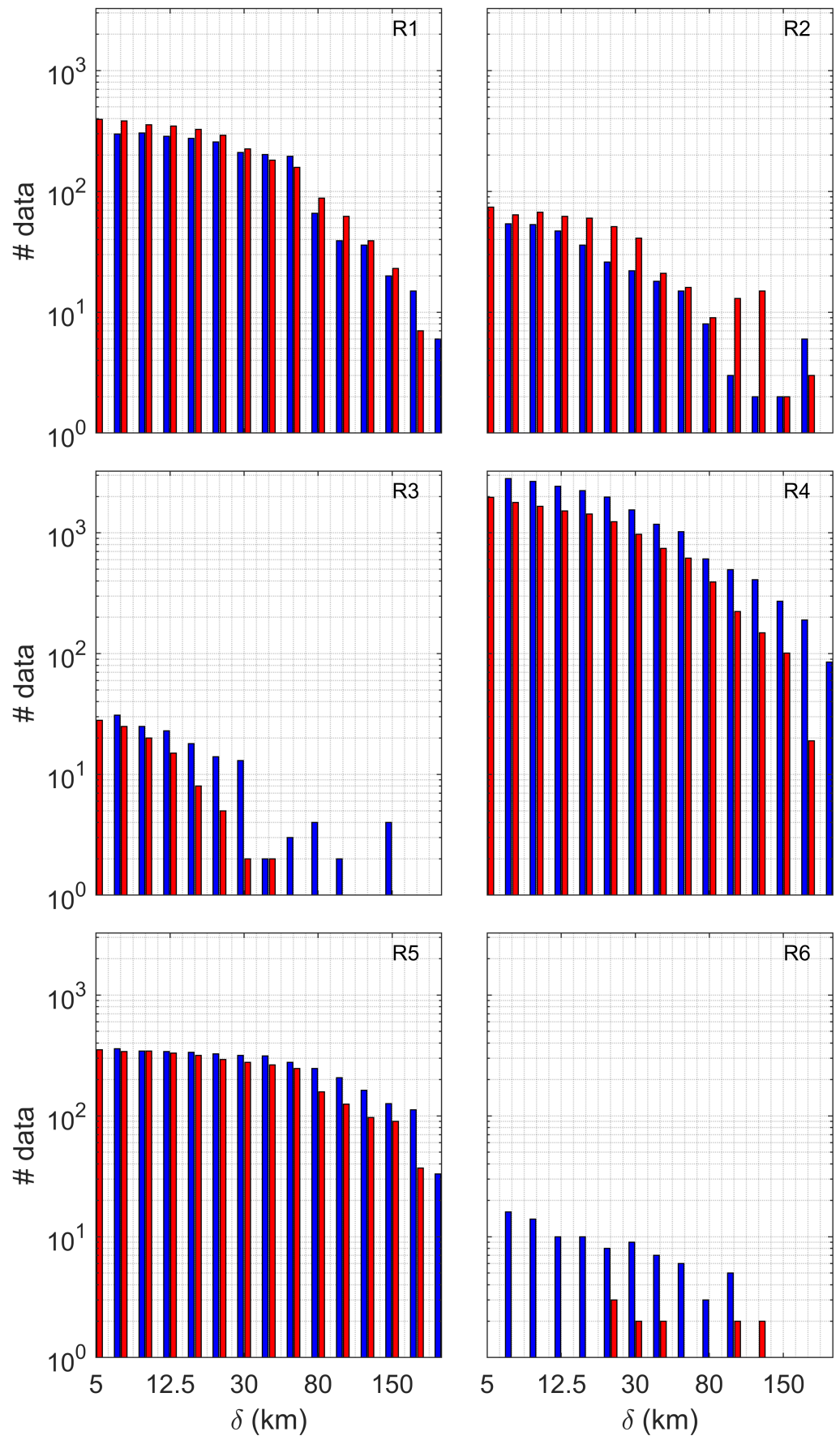

FIG. A3. Number of tracks of pairs of drifters that are inside each SOM-region identified in Fig. 1 at least 747 during 2 days used. 


\section{References}

Abascal, A. J., S. Castanedo, F. J. Mendez, R. Medina, and I. J. Losada, 2009: Calibration of a Lagrangian transport model using drifting buoys deployed during the Prestige oil spill. Journal of Coastal Research, 25 (1 (251)), 80-90.

Ardhuin, F., L. Marié, N. Rascle, P. Forget, and A. Roland, 2009: Observation and estimation of Lagrangian, Stokes, and Eulerian currents induced by wind and waves at the sea surface. Journal of Physical Oceanography, 39 (11), 2820-2838.

Artale, V., G. Boffetta, A. Celani, M. Cencini, and A. Vulpiani, 1997: Dispersion of passive tracers in closed basins: Beyond the diffusion coefficient. Physics of Fluids, 9 (11), 3162-3171.

Aurell, E., G. Boffetta, A. Crisanti, G. Paladin, and A. Vulpiani, 1997: Predictability in the large: an extension of the concept of Lyapunov exponent. Journal of Physics A: Mathematical and General, 30 (1), 1.

Berrisford, P., and Coauthors, 2011: The ERA-Interim archive, version 2.0.

Bettencourt, J. H., C. López, E. Hernández-García, I. Montes, J. Sudre, B. Dewitte, A. Paulmier, and V. Garçon, 2015: Boundaries of the Peruvian oxygen minimum zone shaped by coherent mesoscale dynamics. Nature Geoscience, 8 (12), 937-940.

Boffetta, G., A. Celani, and M. Vergassola, 2000: Inverse energy cascade in two-dimensional turbulence: Deviations from gaussian behavior. Physical Review E, 61 (1), R29.

Boffetta, G., G. Lacorata, G. Redaelli, and A. Vulpiani, 2001: Detecting barriers to transport: a review of different techniques. Physica D: Nonlinear Phenomena, 159 (1-2), 58-70.

Bouzaiene, M., M. Menna, P.-M. Poulain, A. Bussani, and D. Elhmaidi, 2020: Analysis of the surface dispersion in the Mediterranean sub-basins. Frontiers in Marine Science.

Corrado, R., G. Lacorata, L. Palatella, R. Santoleri, and E. Zambianchi, 2017: General characteristics of relative dispersion in the ocean. Scientific Reports, 7 (1), 1-11.

Dobler, D., T. Huck, C. Maes, N. Grima, B. Blanke, E. Martinez, and F. Ardhuin, 2019: Large impact of Stokes drift on the fate of surface floating debris in the South Indian Basin. Marine pollution bulletin, 148, 202-209. 
d'Ovidio, F., V. Fernández, E. Hernández-García, and C. López, 2004: Mixing structures in the Mediterranean Sea from finite-size Lyapunov exponents. Geophysical Research Letters, 31 (17).

Ekman, V. W., 1905: On the influence of the earth's rotation on ocean-currents.

Espa, S., G. Lacorata, and G. Di Nitto, 2014: Anisotropic Lagrangian dispersion in rotating flows with a $\beta$ effect. Journal of physical oceanography, 44 (2), 632-643.

Haller, G., 2015: Lagrangian coherent structures. Annual Review of Fluid Mechanics, 47, 137-162.

Hansen, D. V., and P.-M. Poulain, 1996: Quality control and interpolations of WOCE-TOGA drifter data. Journal of Atmospheric and Oceanic Technology, 13 (4), 900-909.

Haza, A. C., A. C. Poje, T. M. Özgökmen, and P. Martin, 2008: Relative dispersion from a high-resolution coastal model of the Adriatic Sea. Ocean Modelling, 22 (1-2), 48-65.

Hernández-Carrasco, I., E. Alou-Font, P.-A. Dumont, A. Cabornero, J. Allen, and A. Orfila, 2020: Lagrangian flow effects on phytoplankton abundance and composition along filamentlike structures. Progress in Oceanography, 189, 102469.

Hernández-Carrasco, I., C. López, E. Hernández-García, and A. Turiel, 2011: How reliable are finite-size Lyapunov exponents for the assessment of ocean dynamics? Ocean Modelling, $36(3-4), 208-218$.

Hernández-Carrasco, I., C. López, E. Hernández-García, and A. Turiel, 2012: Seasonal and regional characterization of horizontal stirring in the global ocean. Journal of Geophysical Research: Oceans, 117 (C10).

Hernández-Carrasco, I., A. Orfila, V. Rossi, and V. Garçon, 2018: Effect of small scale transport processes on phytoplankton distribution in coastal seas. Scientific reports, 8 (1), 1-13.

Hernández-Carrasco, I., V. Rossi, E. Hernández-García, V. Garçon, and C. López, 2014: The reduction of plankton biomass induced by mesoscale stirring: A modeling study in the Benguela upwelling. Deep Sea Research Part I: Oceanographic Research Papers, 83, 65-80.

Huang, N. E., 1979: On surface drift currents in the ocean. Journal of Fluid Mechanics, 91 (1), 191-208. 
Hui, Z., and Y. Xu, 2016: The impact of wave-induced Coriolis-Stokes forcing on satellitederived ocean surface currents. Journal of Geophysical Research: Oceans, 121 (1), 410-426, https://doi.org/10.1002/2015JC011082.

Janssen, P. A., B. Hansen, and J.-R. Bidlot, 1997: Verification of the ECMWF wave forecasting system against buoy and altimeter data. Weather and Forecasting, 12 (4), 763-784.

Kai, E. T., V. Rossi, J. Sudre, H. Weimerskirch, C. Lopez, E. Hernandez-Garcia, F. Marsac, and V. Garçon, 2009: Top marine predators track Lagrangian coherent structures. Proceedings of the National Academy of Sciences, 106 (20), 8245-8250.

Lacorata, G., E. Aurell, and A. Vulpiani, 2001: Drifter dispersion in the Adriatic Sea: Lagrangian data and chaotic model. Annales Geophysicae, Copernicus GmbH, Vol. 19, 121-129.

Lacorata, G., R. Corrado, F. Falcini, and R. Santoleri, 2019: FSLE analysis and validation of Lagrangian simulations based on satellite-derived GlobCurrent velocity data. Remote sensing of environment, 221, 136-143.

Legrand, T., A. Di Franco, E. Ser-Giacomi, A. Calo, and V. Rossi, 2019: A multidisciplinary analytical framework to delineate spawning areas and quantify larval dispersal in coastal fish. Marine environmental research, 151, 104761.

Lehahn, Y., F. d'Ovidio, M. Lévy, and E. Heifetz, 2007: Stirring of the northeast Atlantic spring bloom: A Lagrangian analysis based on multisatellite data. Journal of Geophysical Research: Oceans, 112 (C8).

Lévy, M., P. J. Franks, and K. S. Smith, 2018: The role of submesoscale currents in structuring marine ecosystems. Nature communications, 9 (1), 1-16.

McWilliams, J. C., and B. Fox-Kemper, 2013: Oceanic wave-balanced surface fronts and filaments. Journal of Fluid Mechanics, 730, 464-490.

McWilliams, J. C., J. Gula, M. J. Molemaker, L. Renault, and A. F. Shchepetkin, 2015: Filament frontogenesis by boundary layer turbulence. Journal of Physical Oceanography, 45 (8), 19882005. 
McWilliams, J. C., E. Huckle, J. Liang, and P. P. Sullivan, 2014: Langmuir turbulence in swell. Journal of physical oceanography, 44 (3), 870-890.

McWilliams, J. C., E. Huckle, J.-H. Liang, and P. P. Sullivan, 2012: The Wavy Ekman Layer: Langmuir Circulations, Breaking Waves, and Reynolds Stress. Journal of Physical Oceanography, 42 (11), 1793 - 1816, https://doi.org/10.1175/JPO-D-12-07.1, URL https://journals.ametsoc.org/view/journals/phoc/42/11/jpo-d-12-07.1.xml.

McWilliams, J. C., and J. M. Restrepo, 1999: The Wave-Driven Ocean Circulation. Journal of Physical Oceanography, 29 (10), 2523 - 2540, https://doi.org/10.1175/1520-0485(1999) 029<2523:TWDOC>2.0.CO;2, URL https://journals.ametsoc.org/view/journals/phoc/29/10/ 1520-0485_1999_029_2523_twdoc_2.0.co_2.xml.

Menna, M., R. Gerin, A. Bussani, and P.-M. Poulain, 2017: The OGS Mediterranean Drifter Dataset: 1986-2016. Rel. OGS, 92.

Morales-Márquez, V., I. Hernández-Carrasco, G. Simarro, V. Rossi, and A. Orfila, 2020: Regionalizing the impacts of wind and wave-induced currents on surface ocean dynamics: a long-term variability analysis in the Mediterranean Sea. Essoar, https://doi.org/https: //doi.org/10.1002/essoar.10505583.1.

Obermann, A., S. Bastin, S. Belamari, D. Conte, M. A. Gaertner, L. Li, and B. Ahrens, 2018: Mistral and Tramontane wind speed and wind direction patterns in regional climate simulations. Climate Dynamics, 51 (3), 1059-1076, https://doi.org/https://doi.org/10.1007/s00382-016-3053-3.

Onink, V., D. Wichmann, P. Delandmeter, and E. Van Sebille, 2019: The role of Ekman currents, geostrophy, and stokes drift in the accumulation of floating microplastic. Journal of Geophysical Research: Oceans, 124 (3), 1474-1490.

Polton, J. A., D. M. Lewis, and S. E. Belcher, 2005: The role of wave-induced Coriolis-Stokes forcing on the wind-driven mixed layer. Journal of Physical Oceanography, 35 (4), 444-457.

Price, J. F., R. A. Weller, and R. R. Schudlich, 1987: Wind-Driven Ocean Currents and Ekman transport. Science, 238 (4833), 1534-1538, https://doi.org/10.1126/science.238.4833.1534. 
Richardson, L. F., 1926: Atmospheric diffusion shown on a distance-neighbour graph. Proceedings of the Royal Society of London. Series A, Containing Papers of a Mathematical and Physical Character, 110 (756), 709-737.

Rossi, V., E. Ser-Giacomi, C. López, and E. Hernández-García, 2014: Hydrodynamic provinces and oceanic connectivity from a transport network help designing marine reserves. Geophysical Research Letters, 41 (8), 2883-2891, https://doi.org/https://doi.org/10.1002/2014GL059540.

Santiago-Mandujano, F., and E. Firing, 1990: Mixed-layer shear generated by wind stress in the central equatorial Pacific. Journal of Physical Oceanography, 20 (10), 1576-1582.

Sayol, J. M., A. Orfila, and L.-Y. Oey, 2016: Wind induced energy-momentum distribution along the Ekman-Stokes layer. Application to the Western Mediterranean Sea climate. Deep Sea Research Part I: Oceanographic Research Papers, 111, 34-49.

Sayol, J. M., A. Orfila, G. Simarro, D. Conti, L. Renault, and A. Molcard, 2014: A Lagrangian model for tracking surface spills and SaR operations in the ocean. Environmental Modelling \& Software, 52, 74-82.

Ser-Giacomi, E., G. Jordá-Sánchez, J. Soto-Navarro, S. Thomsen, J. Mignot, F. Sevault, and V. Rossi, 2020: Impact of climate change on surface stirring and transport in the Mediterranean Sea. Geophysical Research Letters, 47 (22), e2020GL089 941.

Ser-Giacomi, E., T. Legrand, I. Hernández-Carrasco, and V. Rossi, 2021: Explicit and implicit network connectivity: Analytical formulation and application to transport processes. Phys. Rev. E, 103, 042 309, https://doi.org/10.1103/PhysRevE.103.042309, URL https://link.aps.org/doi/ 10.1103/PhysRevE.103.042309.

Shadden, S. C., F. Lekien, and J. E. Marsden, 2005: Definition and properties of lagrangian coherent structures from finite-time Lyapunov exponents in two-dimensional aperiodic flows. Physica D: Nonlinear Phenomena, 212 (3-4), 271-304.

Small, R., S. Carniel, T. Campbell, J. Teixeira, and R. Allard, 2012: The response of the Ligurian and Tyrrhenian Seas to a summer Mistral event: A coupled atmosphere-ocean approach. Ocean Modelling, 48, 30-44. 
Soukissian, T., F. Karathanasi, P. Axaopoulos, E. Voukouvalas, and V. Kotroni, 2018: Offshore wind climate analysis and variability in the Mediterranean Sea. International Journal of Climatology, 38 (1), 384-402.

Sudre, J., C. Maes, and V. Garçon, 2013: On the global estimates of geostrophic and Ekman surface currents. Limnology and Oceanography: Fluids and Environments, 3 (1), 1-20, https://doi.org/ https://doi.org/10.1215/21573689-2071927.

Suzuki, N., and B. Fox-Kemper, 2016: Understanding Stokes forces in the wave-averaged equations. J. Geophys. Res. Oceans, 121, 3579-3596.

Taylor, G. I., 1921: Experiments with rotating fluids. Proceedings of the Royal Society of London. Series A, Containing Papers of a Mathematical and Physical Character, 100 (703), 114-121.

Van Sebille, E., and Coauthors, 2018: Lagrangian ocean analysis: Fundamentals and practices. Ocean Modelling, 121, 49-75.

Welander, P., 1957: Wind Action on a Shallow Sea: Some generalizations of ekman's theory. Tellus, 9 (1), 45-52, https://doi.org/https://doi.org/10.1111/j.2153-3490.1957.tb01852.x.

Wenegrat, J. O., and M. J. McPhaden, 2016: Wind, waves, and fronts: Frictional effects in a generalized Ekman model. Journal of Physical Oceanography, 46 (2), 371-394, https://doi.org/10.1175/ JPO-D-15-0162.1, URL https://journals.ametsoc.org/view/journals/phoc/46/2/jpo-d-15-0162. 1.xml.

Zecchetto, S., and F. De Biasio, 2007: Sea surface winds over the Mediterranean basin from satellite data (2000-04): Meso-and local-scale features on annual and seasonal time scales. Journal of Applied Meteorology and Climatology, 46 (6), 814-827, https://doi.org/DOI:10.1175/JAM2498. 1. 